\title{
Aménagement des Terres Incultes et Émancipation des Femmes L'Expérience de SARTHI
}

Madhu Sarin

Follow this and additional works at: https://knowledgecommons.popcouncil.org/departments_sbsr-pgy

Part of the Agricultural and Resource Economics Commons, Family, Life Course, and Society

Commons, Gender and Sexuality Commons, Inequality and Stratification Commons, and the International

Public Health Commons

How does access to this work benefit you? Let us know!

\section{Recommended Citation}

Sarin, Madhu. 1993. "Aménagement des Terres Incultes et Émancipation des Femmes L'Expérience de SARTHI," SEEDS no. 16. New York: Population Council. 


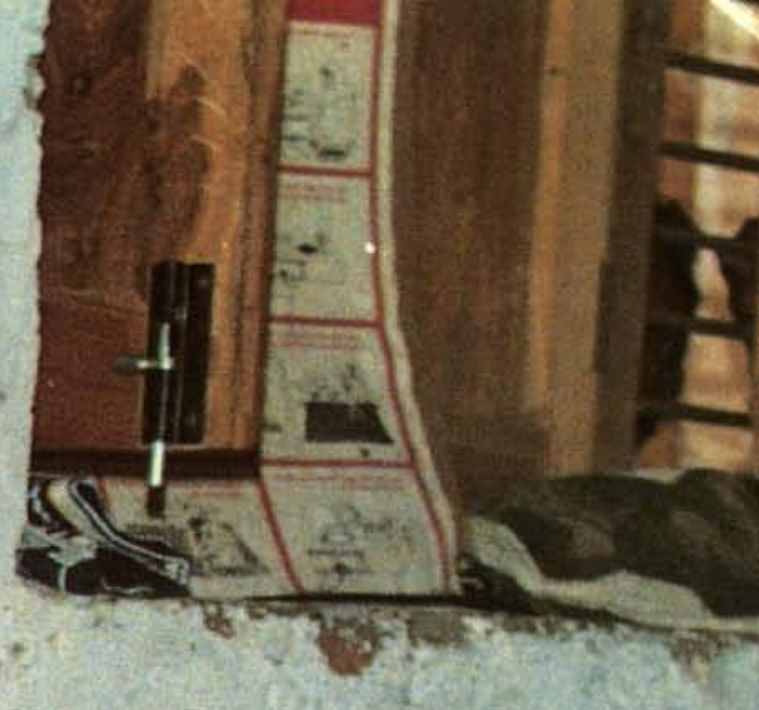

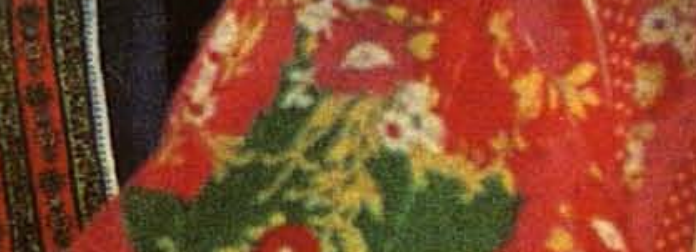

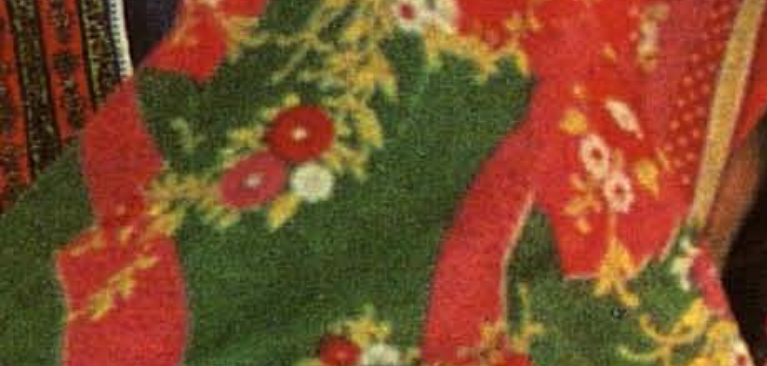

? 3 का जा जो $x^{2}=x^{2} \times 1$

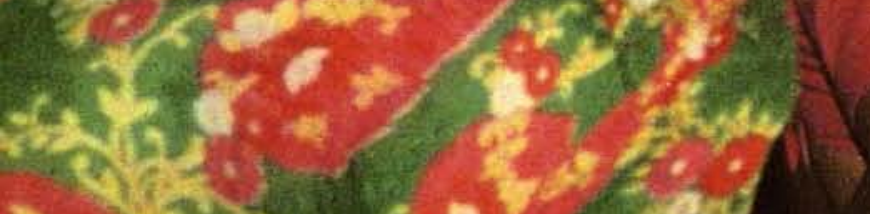

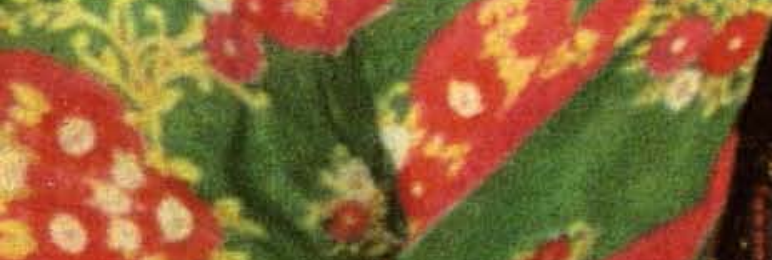
50 d $x^{2}+2=2$ $3 x^{\circ}+1$ $-x^{2}$ है

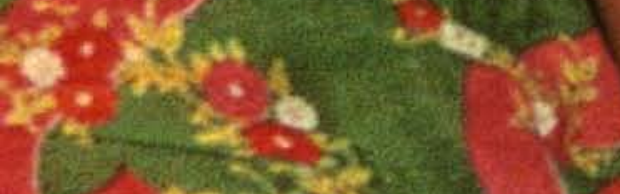

C.F.

$1-2$

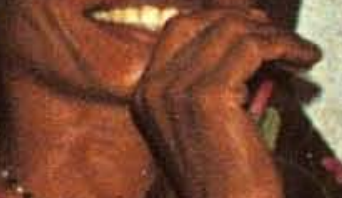
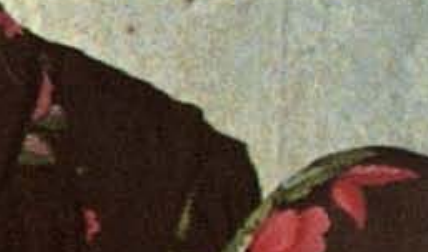
SEEDS est une série de brochures publiée en réponse aux demandes d'information venant du monde entier sur des idées de programmes innovatrices et pratiques, développées pour aborder le problème de la condition et des besoins économiques des femmes aux revenus faibles. Le but de ces brochures est de diffuser l'information et de stimuler la création de nouvelles initiatives fondées sur les expériences positives de projets visant à aider les femmes à gagner leur vie et améliorer leur statut économique. Les projets décrits dans ce numéro comme dans le reste de la série ont été choisis du fait qu'ils servent non seulement à renforcer les rôles producteurs des femmes mais aussi à assurer leur intégration dans différents secteurs de développement à la fois sur le plan économique et social. Tous les projets publiés dans le cadre de la série SEEDS associent les femmes aux prises de décision, organisent les femmes au niveau local et abordent les problèmes de politique générale qui influent sur les rôles économiques des femmes.

Ces rapports n'ont cependant pas été conçus pour être suivis à la lettre car chaque effort de développement doit faire face à des problèmes et des possibilités quelque peu différents. En revanche, ils relatent l'histoire d'une idée et sa mise en application avec l'espoir que les leçons apprises se révéleront utiles dans des environnements variés. C'est également pour être portés à l'attention de ceux qui détiennent les pouvoirs de décision qu'ils ont été rédigés et leur montrer que les femmes jouent des rôles vitaux non seulement dans l'économie de leurs ménages mais également dans la vie économique de chaque nation.

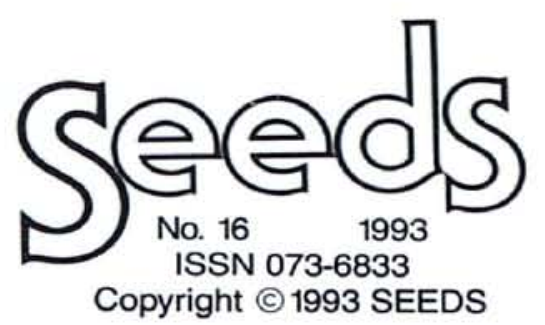

La publication de SEEDS est administrée par le Population Council. Les critères de publication sont déterminés par le Comité de Rédaction de SEEDS: Judith Bruce (Population Council), Betsy Campbell (The Ford Foundation), Marilyn Carr (UNIFEM), Marty Chen (Harvard Institute for International Development), Anne Kubisch (The Ford Foundation), Ann Leonard (The Population Council), Cecilia Lotse (UNICEF), Katharine McKee (Center for Community SelfHelp), Anne Walker (International Women's Tribune Center) et Mildred Warner (Cornell University).

La traduction française de ce numéro de SEEDS a été subventionnée par la Agence canadienne de développement international (CIDA).

Les opinions et commentaires exprimés dans cette brochure sont la seule responsabilité des auteurs et n'engagent qu'eux. 


\section{Aménagement \\ des Terres Incultes et \\ Émancipation des Femmes \\ L'Expérience de SARTHI}

par Madhu Sarin

\section{Introduction}

Pour de larges segments de la population rurale de l'Inde, l'agriculture de subsistance constitue la principale ressource économique. Pourtant, même au cours des années où il pleut suffisamment, la production agricole ne suffit pas à couvrir l'ensemble des besoins des ménages puisque la plupart des familles ne disposent d'aucun revenu en espèces. C'est la raison pour laquelle, les familles s'adonnent également à la collecte de produits végétaux (que l'on désignera ci-après par "biomasse") comme, par exemple, le combustible, le bois pour la construction, le fourrage, les plantes et les racines médicinales.

Cette activité de collecte de la biomasse est presque exclusivement effectuée par les femmes. Dès lors, les femmes ont un intérêt tout particulier à sauvegarder les ressources naturelles locales puisque ce sont elles qui souffrent le plus des conséquences de la dégradation du sol et du déboisement. Mais se contenter d'impliquer les femmes dans des activités de plantation d'arbres, d'arbustes et d'herbage n'est pas une solution adéquate puisque les femmes n'ont pas le droit de posséder des terres et n'ont aucun contrôle sur les ressources et les produits de la terre. A moins que les projets de développement ne permettent aux femmes d'obtenir davantage de contrôle sur l'utilisation et l'exploitation des ressources locales, il est peu probable qu'elles bénéficieront des efforts de remise en valeur de la terre de façon durable.

Ce numéro de SEEDS est consacré à une approche innovative ayant pour but d'aménager des terres incultes. Cette initiative a été développée par SARTHI (Action Sociale en faveur des population rurales et autochtones d'Inde), une organisation non gouvernementale basée dans le district de Panchmahals dans l'Etat de Gujarat en Inde occidentale. En aidant les femmes rurales à s'organiser pour mettre en valeur des lopins de terres communaux, SARTHI a permis à ces femmes de satisfaire leurs besoins en biomasse de façon plus efficace et saine d'un point de vue écologique et d'améliorer d'autres aspects de leur vie.

L'auteur aimerait dédié ce numéro de SEEDS à la mémoire de Champa Ben, une habitante du village de Godhar où se trouve les bureaux de SARTHI. De villageoise timide et craintive, qu'on a amené à force de cajoleries à participer au programme de formation de PEDO pour devenir constructrice de fourneau, Champa Ben est devenue une militante et une monitrice étonnante et attentive. En compagnie de Vikram Bhai, son collègue de sexe masculin, elle a contribué à fonder le premier groupe de femmes à Muvasa. Malheureusement, Champa Ben est morte des suites d'un cancer de l'utérus. Elle avait trente ans à peine. Même à travers sa mort, elle aura sensibilisé les femmes locales sur la nécessité d'aller se faire examiner régulièrement pour réduire l'incidence des décès dus à de telles maladies. 


\section{Les Terres Incultes d'Inde}

Sur les quelque 300 millions d'hectares de terre du pays, seul environ $\mathbf{4 0}$ millions ne sont pas arables. Et sur les 260 millions d'hectares de terres cultivables, environ deux tiers appartiennent à des particuliers. L'autre tiers consiste en ce que l'on appelle des "terres communales" qui sont sous le contrôle de l'Etat ou d'institutions locales. Entre 30 et 50 pour cent de l'ensemble des terres aussi bien privées que communales sont érodées à des degrés divers. En Inde, on appelle ce genre de terre "terre inculte", et on entend par là toute terre qui n'est pas en mesure de produire son potentiel de biomasse parce que érodée, surexploitée ou mal gérée.

En Inde, les populations rurales pauvres en terres et en disponibilités dépendent exclusivement des terres communales pour s'approvisionner en biomasse. En raison de l'inquiétude croissante sur la dégradation rapide des ressources naturelles du pays, l'état a lancé un programme massif pour remettre en valeur les terres incultes.

\section{Le District de Panchmahals}

Les forêts du Panchmahals ont été célébres tout au long de l'histoire de l'Inde. C'est là que les sultans d'Ahmdedabad et les empereurs Mughal se rendaient pour chasser des éléphants. Malheureusement, ces forêts ont presque entièrement disparu essentiellement à cause de l'abattage illégal d'arbres pour la construction et en raison de l'exploitation commerciale par les souverains princiers puis, par le gouvernement local après l'indépendance. Le défrichement, d'une part, et le pâturage incontrollé d'animaux domestiques, d'autre part, ont également contribué massivement à ce déboisement. Le pâturage excessif est particulièment nuisible puisqu'il tend à détruire le processus de régénération naturelle. De surcroit, chaque année de sécheresse ne fait qu'aggraver la situation. En effet, pour compenser les mauvaises récoltes, la population locale désespérée se tourne vers la forêt pour obtenir le fourrage et le bois dont elle a besoin.

Les sécheresses les plus récentes ont eu lieu entre 1985 et 1987 et ont plongé les gens de la région dans la misère. En plus de l'absence totale de récolte, il y avait également une pénurie de fourrage ce qui a provoqué la mort de nombreux animaux. Dans une région où la migration saisonnière est déjà élevée (étant donné que l'agriculture ne produit qu'une récolte par an), un nombre encore plus important de personnes se sont vues contraintes de migrer vers d'autres régions pour trouver du travail.

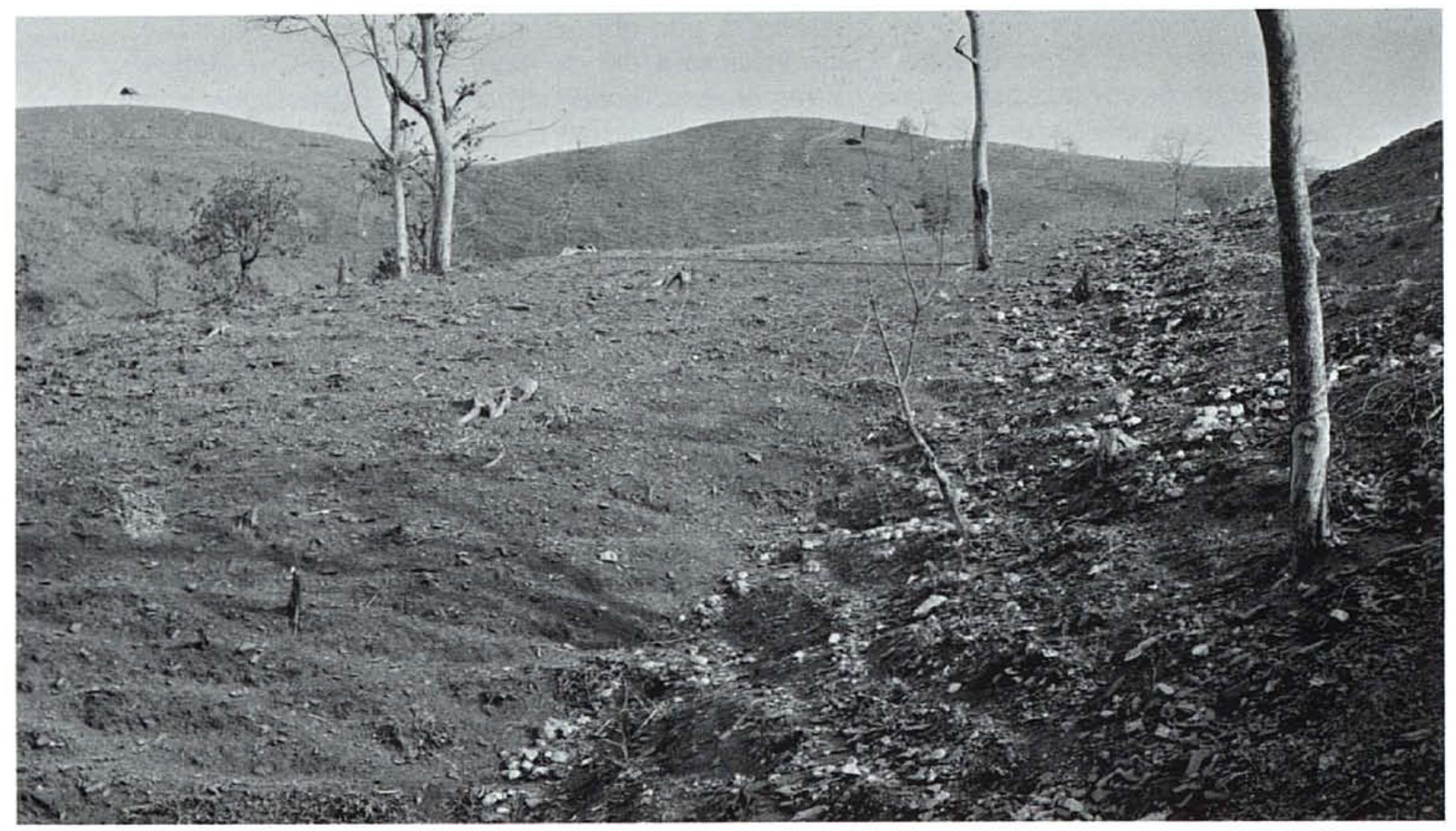


Le district de Panchmahals est l'un des moins industrialisés de tout l'état de Gujarat avec $89 \%$ des habitants travaillant dans le secteur primaire. La plupart d'entre eux gagnent leur vie comme petits cultivateurs marginaux. 90 pour cent de toutes les terres cultivées dans le district sont nourries par la pluie ce qui rend la région particulièrement vulnérable aux conditions climatiques. Par rapport à l'ensemble de l'Etat de Gujarat, le district de Panchmahals a une densité de la population plus forte, un taux d'alphabétisation plus faible (plus particulièrement parmi les femmes), une urbanisation moins avancée et une proportion plus forte d'Adivasis c'est-à-dire de population autochtone.

Les Adivasis, considérés comme les habitants indigènes de l'inde, représentent environ 7 pour cent de la population à l'échelon national. A Santrampur taluka (une sous-unité administrative du district de Panchmahals) où SARTHI est implanté, 66 pour cent de la population est d'origine Adivisi alors qu'elle n'est que de 14 pour cent pour l'ensemble de l'état de Gujarat.

Les Adivasis vivent essentiellement dans la forêt, plus particulièrement dans la région forestière du centre et de l'est de l'Inde. Ils sont composés d'un nombre important de tribus différentes ayant chacune leur propre culture, identité et langue. Bien qu'ils demeurent dans des régions riches en ressources naturelles, ils n'ont pas vraiment été touchés par le "développement".

Les anglais ont introduit plusieurs mesures pour préserver l'identité culturelle et les intérêts économiques des Adivasis. Après l'Indépendence, le gouvernement d'Inde a également instaurer des mesures législatives et administratives pour protéger leurs intérêts. Un "inventaire" ou liste des tribus reconnues donne droit à ceux qui appartiennent à l'une des "tribus reconnues" à certains bénéfices et services comme, par exemple, l'accès à des emplois gouvernementaux ou à des sièges dans les instituts d'éducation supérieure spécialement réservés pour eux ou encore l'octroi de prêts spéciaux ou de programmes de subventions. Dans les régions où plus de 50 pour cent de la population appartient à des tribus reconnues, des plans de développement spéciaux sont établis en faveur des tribus.

La législation proscrit l'achat de terre appartenant à un Adivasi par un non-adivasi. Toutefois, en dépit de toutes ces mesures de protection, la marginalisation de ces tribus n'a fait que s'accroitre au lieu de diminuer. Des projets de développement massifs, comme la construction de barrages, de centrales électriques ou d'aciéries ont forcé un nombre plus important

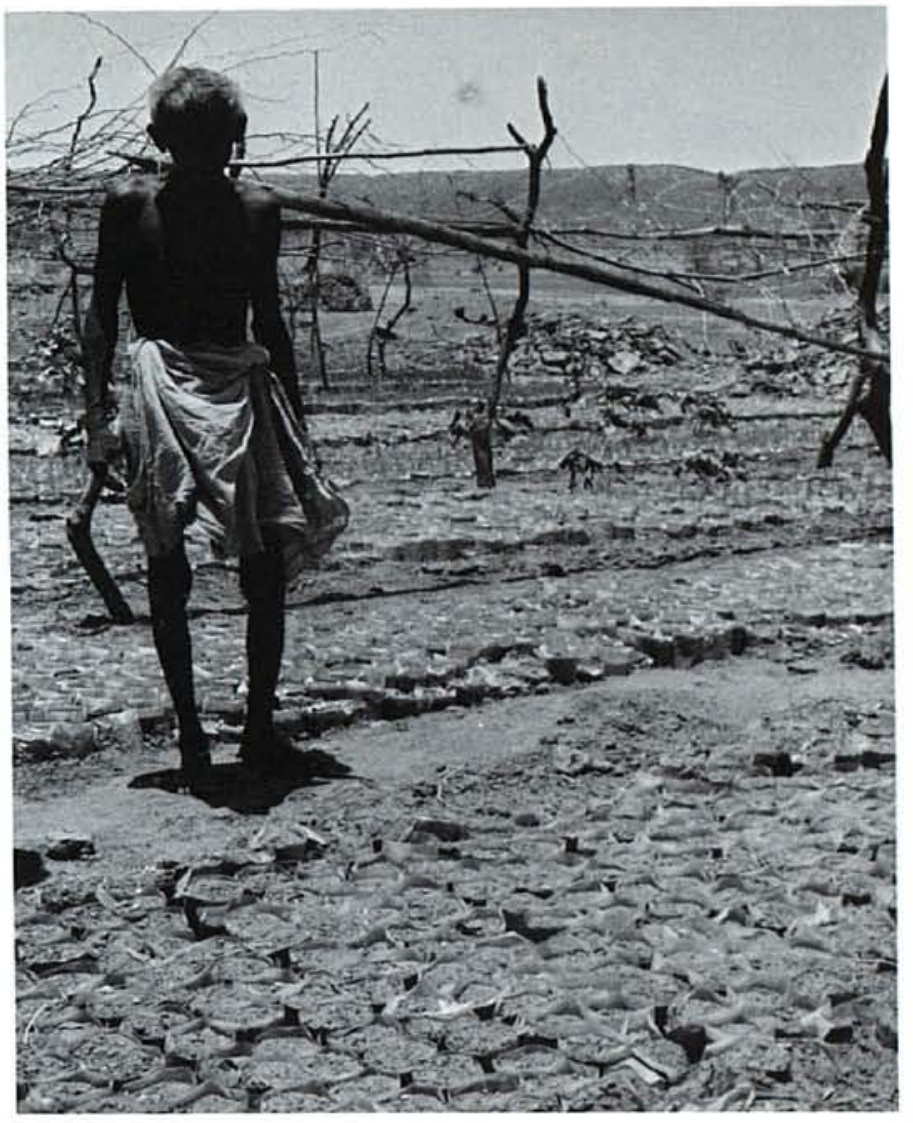

d'Adivasis de quitter leur habitat traditionnel que d'autres segments de la population. Par ailleurs, la destruction des forêts à cause de l'exploitation commerciale ainsi que d'autres projets de développement ont eu pour effet de ruiner l'économie des Adivasis-essentiellement basée sur la cueillette et la chasse dans les forêts-sans pour autant leur procurer une source de revenue alternative et stable.

\section{La Gestion des Terres Incultes}

Dans l'ensemble de I'Inde, une proportion significative de terres est sous le contrôle du gouvernement. Par exemple, environ 22 pour cent de l'ensemble des terres communales sont contrôlées par le Département Forestier de l'Etat. Dans la région de Panchmahals où SARTHI est implanté, le Département Forestier contrôle 16 pour cent des terres et le Fisc neuf pour cent. Les terres contrôlées par le Fisc sont appelées "terres incultes fiscales". Par ailleurs, environ 4 pour cent de l'ensemble des terres du pays sont contrôlées par les panchayats (la plus petite unité de gouvernement local regroupant un ou plusieurs villages). Ces terres sont destinées à servir de pâturages communaux. Sur les quelque 50000 hectares de pâturages disponibles dans le district, on estime qu'au moins 10000 hectares ont été empiétés par des particuliers. Le reste est dans un état érodé et improductif en raison de l'ab- 


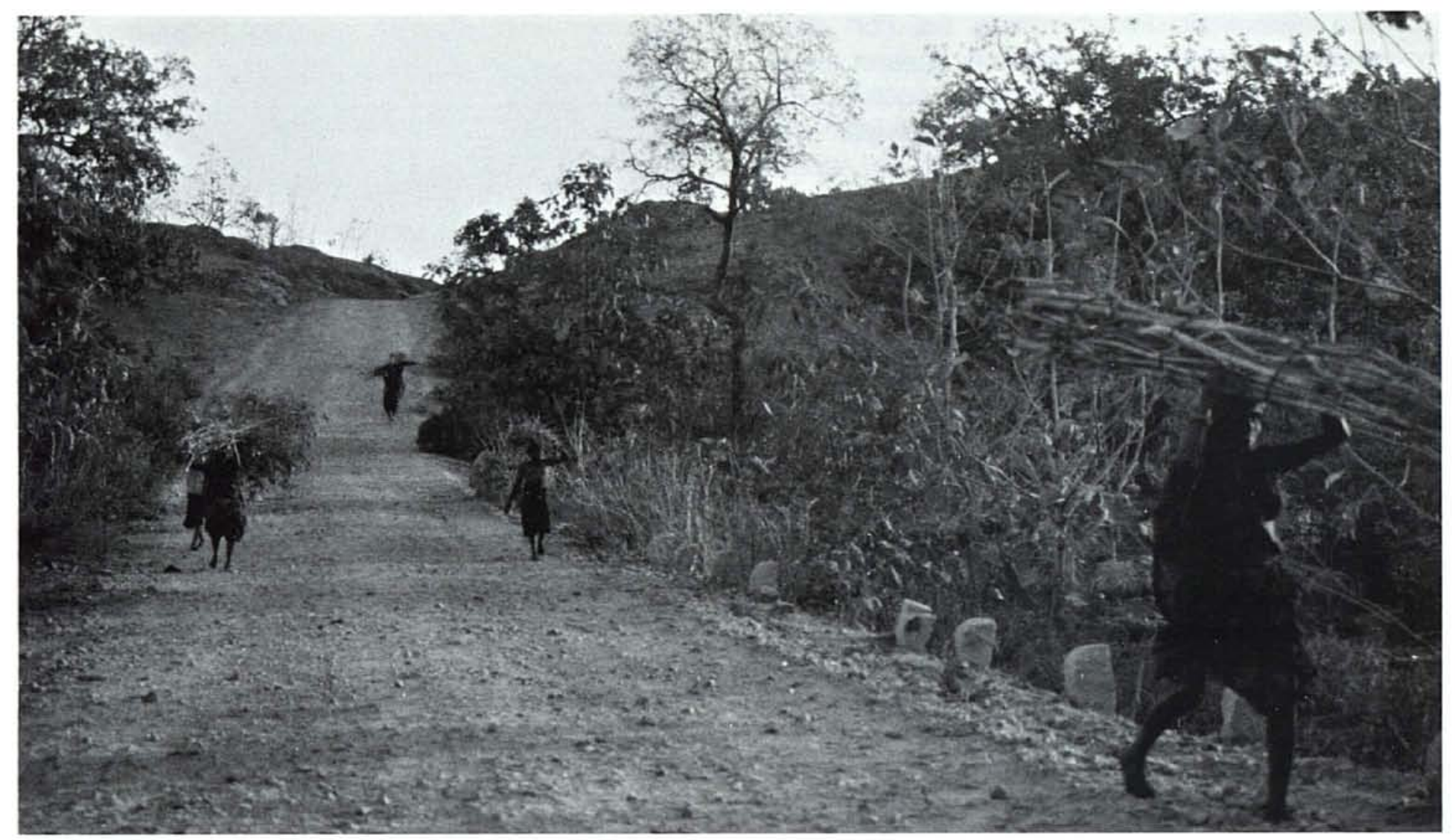

sence d'un système de régulation ou de gestion spécifique.

Etant donné que les fermes de la région sont très petites et que le rendement agricole est insuffisant pour subvenir aux besoins des fermiers, ces derniers augmentent leur revenus en se rendant sur les terres communales environnantes pour amasser de la biomasse. Le déclin des terres communales disponibles et la dégradation continuelle des terres restantes rend cette tâche de plus en plus difficile. Et comme la collecte de biomasse de subsistance est une tâche de femme, ce sont les femmes qui souffrent le plus des conséquences de cette dégradation.

\section{Statut des Femmes et Leur Rapport avec les Ressources Naturelles.}

A Panchmahals, comme dans nombre d'autres régions de l'Inde, le statut des femmes est bas alors que leur charge de travail est lourde. En plus des corvées domestiques, de nombreuses femmes travaillent comme journalière dans les champs des gros propriétaires fonciers ou pour des projets de travaux publics. Les femmes issues de familles qui possèdent des terres remplissent également des tâches agricoles substantielles. Tandis que les hommes labourent et vendent les produits de la récolte sur le marché, les femmes sont responsables du binnage, du sarclage, de l'irrigation, de la moisson et du traitement des céréales. En outre, elles doivent aussi chercher du bois, puiser de l'eau et s'occuper du bétail. Ces activités les lient bien plus à leur environnement naturel que les hommes. Ce sont les femmes qui souffrent le plus des conséquences de la dégradation de l'environnement puisqu'elles sont obligées de parcourir de plus grandes distances pour trouver le bois, le fourrage et l'eau dont elles ont besoin. Comme plusieurs femmes de la région ont noté, "Les hommes s'attendent à être servis. Ils ne se soucient guère de savoir d'où provient le bois. C'est notre problème".

Pour pallier au déboisement, le Département Forestier a commencé par lever des restrictions sur le type de bois que les habitants de la région peuvent collecter et l'usage qui peut en être fait. Par exemple, il est strictement interdit de couper du bois vert pour s'en servir comme combustible. Malheureusement, le bois sec et mort qu'il est possible de ramasser ne se trouve pas en quantité suffisante. Les femmes se voient donc souvent obligées d'enfreindre les restrictions et de couper des branches vertes. Si un garde forestier surprend une femme en train de couper ou de transporter du bois vert, soit il lui prend sa faucille ou sa hache, soit lui confisque son chargement, ou bien lui soutire de l'argent ou encore l'insulte ou la 
maltraite. Etant donné que les gardes-forestier sont tous des hommes, la peur qui hante le plus les femmes c'est de se faire battre ou brutaliser.

L'abandon, la violence domestique et l'alcooholisme (en dépit de l'interdiction d'en consommer dans l'état de Gujarat) parmi les hommes ainsi que le fait que les maris prennent une seconde épouse dans le but d'engendrer un fils sont des problèmes courants pour les femmes de la région. En outre, au cours des années de sécheresse, les femmes sont très souvent obligées d'assumer l'intégralité des travaux agricoles lorsque les hommes migrent à la recherche d'un emploi rémunéré. Quelquefois aussi, les femmes sont obligées de partir avec leur mari pour trouver du travail dans des régions plus prospères.

Les femmes de Panchmahals comme dans le reste de l'Inde possèdent très peu de terres ou de biens immobiliers. Et les décisions relatives à l'usage de la terre sont prises par les hommes. Cela rend donc la remise en valeur des terres par les femmes extrêmement difficile.

\section{Evolution du Programme en Faveur des Femmes de SARTHI}

SARTHI a commencé à travailler dans la région de Santrampur il y a douze ans environ. A l'origine, c'était une filiale du Social Work and Research Centre (Centre d'Assistance Sociale et de Recherche ou SWRC), une ONG basée à Tilonia dans l'Etat de Rajasthan. Mais six ans plus tard, c'est devenu une organisation indépendante. La mission de SARTHI consiste à promouvoir le développement rural intégré dans les régions sous-développées en améliorant la qualité de l'assistance fournie par les ONGs locales. Initialement, SARTHI s'occupait de projets de développement conventionnels, comme par exemple, d'installer des pompes manuelles, de creuser des puits plus profonds et de lancer des activités rémunératrices. L'expérience acquise au fil des ans a conduit SARTHI à modifier ses orientations. L'organisation se consacre désormais exclusivement à la formation de dirigeants et à la promotion d'actions organisées parmi les groupes défavorisés de la société, notamment les femmes.

Le programme en faveur des femmes de SARTHI s'est développé autour d'une série d'initiatives prises pour former des villageoises aux trois corps de métiers suivants : constructeurs de fourneau (chula mistris), agents de santé et paravets (technicien en science vétérinaire).

Lorsque SARTHI a lançé son programme d'installation de fourneau plus performants en 1983, c'était la première fois que l'organisation travaillait exclusivement avec des femmes. Des villageoises préalablement sélectionnées ont suivi une formation pour construire et installer des four-

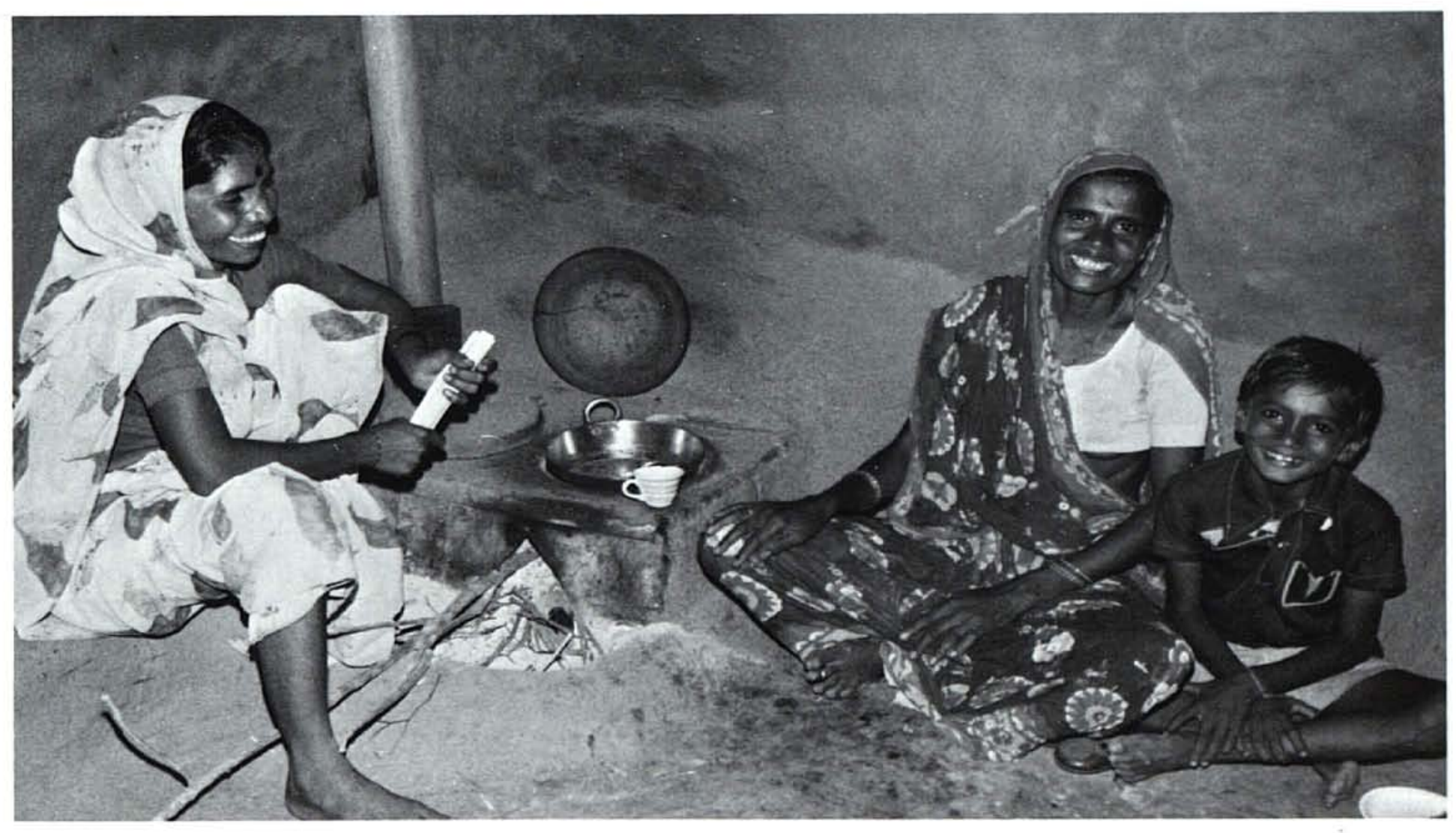


neaux plus performants dans et autour de leurs villages. Quatre ans plus tard, en 1987, trente constructrices et quatre monitrices étaient opérationnelles au niveau des villages. Les quatre monitrices sont devenues les premiers employés locaux féminins à plein temps de SARTHI. Comme chaque constructrice de fourneau était en contact avec un minimum de 30 à 50 femmes, SARTHI s'est trouvé à la tête d'un réseau important de femmes locales. Les réunions qui se tenaient régulièrement tous les mois entre constructrices et monitrices ont permis à l'organisation de mieux comprendre les problèmes auxquels étaient confrontés les femmes de la région.

En juillet 1988, SARTHI a démarré un programme de formation à l'intention des dais (sagefemmes, à la fois traditionnelles et modernes) en réponse à de nombreuses demandes de la part des villageoises d'avoir accès à des services de santé de meilleure qualité. Ces agents de santé femmes étaient le deuxième groupe de villageoises à bénéficier d'une formation. Leur rôle est d'assurer le suivi des femmes qui ont des problèmes de santé et d'orienter les cas qui dépassent leurs compétences vers les services publics de la région.

Puis en mai 1990, de nouveau en réponse à un besoin articulé par les femmes locales, 10 villageoises ont suivi un programme de formation pour devenir paravets. Cela portait donc les groupes de villageoises qualifiées au nombre de trois. En l'espace de deux mois à peine suivant leur formation, ces femmes paravets avaient réussi à vacciner presque 3,000 bestiaux contre la fièvre aphteuse.

Chacun de ces programmes a donné aux femmes l'occasion de se rassembler dans un forum autre que celui de la famille traditionnelle et a permis à SARTHI d'apprendre de première main quels sont les problèmes et les préoccupations des femmes. En outre, ces programmes clairement démontrent que les femmes sont tout à fait capables de s'organiser et d'opérer des changements.

\section{Faire Chaque Chose en son Temps}

Lorsque en 1987 pour la troisième année consécutive il n'y a pas eu de mousson, la situation des populations locales devint alarmante. Des histoires déchirantes de bétail mourrant, de femmes essayant désespérément de trouver du bois, du fourrage, de la nourriture et de l'eau et de maladies décimant des pans entiers de la popula- tion commençaient à circuler de toutes parts. Pour la première fois, SARTHI envisagea sérieusement d'élaborer une approche plus holistique pour répondre aux besoins des femmes.

En avril 1987, SARTHI a décidé d'envoyer un groupe de villageoises et les quatre monitrices à PEDO', une ONG soeur basée à Rajasthan, pour assister à une large mela de femmes (litéralement une foire mais peut se référer à tout rassemblement important de personnes dans un but spécifique). L'objectif de la mela était de permettre aux fermmes locales de discuter et de partager leurs problèmes et d'apprendre par les efforts menés par leurs soeurs dans d'autres régions.

Les femmes sont revenues enthousiastes et passionées à l'idée de s'organiser pour passer à l'action. En novembre 1987, les quatre monitrices ont participé à un stage de formation spécifiquement conçu à l'intention des membres du personnel femmes à PEDO. En plus de partager leur expérience en temps que femme, les participantes exploraient également les possibilités que leur offrait l'action collective pour résoudre un certain nombre de leurs problèmes. Pour la première fois, les femmes étaient appelées à réfléchir sur la crise de l'environnement-ses causes et ce que les femmes pouvaient faire pour renverser la tendance.

L'objectif était d'amener les femmes à examiner la nature des changements écologiques qui se sont produits au cours des dernières années, en déterminant notamment les causes et l'impact que ces changements ont eu sur leur vie quotidienne. Ce travail d'analyse s'est avéré très révélateur pour les femmes. Jamais auparavant, n'avaient elles abordé le problème d'une manière aussi totale. Pour la première fois, elles ont réalisé combien les arbres et la forêt étaient indispensables à leur survie. Qu'il s'agissait d'une baisse de la productivité du sol, de chutes de pluie incertaines ou en diminution, du déclin du niveau hydrostatique, du tarissement des sources d'eaux perpétuelles, de la pénurie de combustible, de fourrage et d'aliments nutritifs, ou encore d'une recrudescence des problèmes de santé, tous ces problèmes pouvaient être attribués dans une certaine mesure à la destruction de la forêt.

\footnotetext{
1 PEDO était initialement aussi une filiale du SRWC et sa mission est identique à celle de SARTHI. PEDO se situe à environ trois heures de route de SARTHI. En raison des liens étroits qui existent entre les directeurs des deux ONGs et de la similarité de leurs programmes, chaque groupe fréquemment participe aux activités organisées par l'autre.
} 


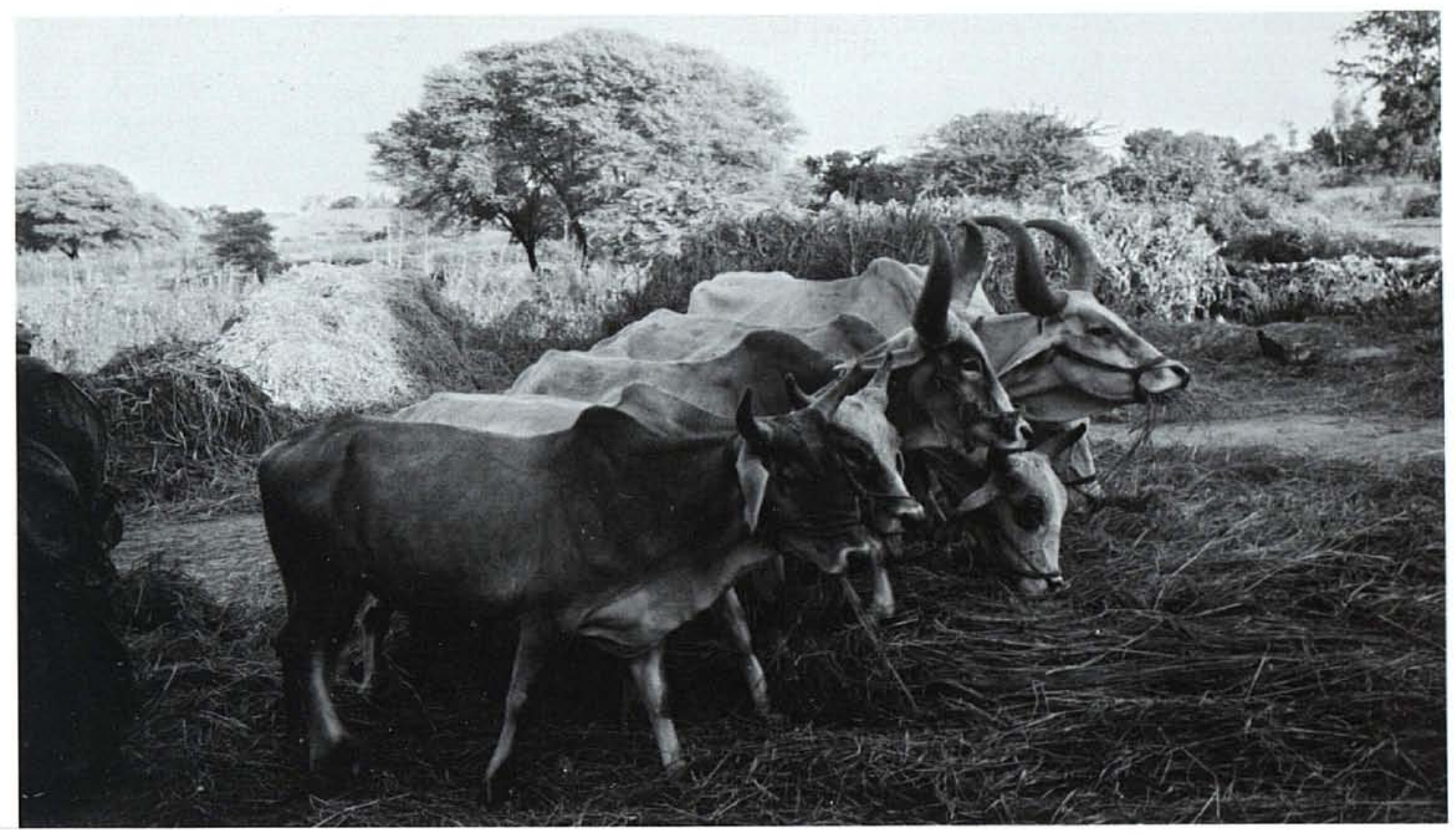

Une fois ce travail de réflexion achevé, les femmes se sont mises en petits groupes pour procéder à un exercice de planification. On leur a demandé d'évaluer le nombre d'arbres et d'arbustes qui seraient nécessaires à chaque membre du groupe pour satisfaire ses besoins en combustible, fourrage, bois de construction et autres nécessités et d'établir une liste des espèces d'arbres locales appropriées à chaque usage. Cet exercice a suscité un intérêt considérable chez les femmes puisqu'il leur fournissait une base concrète pour discuter des stratégies d'action avec d'autres femmes.

Par exemple, les femmes ont calculé que s'il fallait nourrir les vaches ou les boeufs exclusivement à base de feuilles d'arbres, 90 arbres de grande taille seraient nécessaires pour nourrir un animal pendant une année. Pour une chèvre ou un mouton, il faudrait compter 50 arbres. Les femmes ont identifié 21 espèces locales pouvant servir de fourrage. Des calculs similaires ont été établis pour déterminer la quantité de bois nécessaire pour construire une maison de deux pièces. D'après leurs estimations, cinq à six arbres de grande taille ou 150 arbres plus petits seraient nécessaires. De plus, il leur faudrait également 100 tiges de bambous pour la charpente et environ 200 à 300 kilos de bois pour cuire les tuiles. Les femmes étaient vraiment surprises par la quantité d'arbres qu'il leur fallait rien que pour satisfaire leurs besoins les plus élementaires.

SARTHI n'avait pas encore à ce moment là établi de programme spécifique pour faire participer les femmes à la remise en valeur des terres incultes. Après leur stage à PEDO, les quatre monitrices ont commençé à aborder avec les villageoises le problème de la gestion des ressources naturelles au cours de leurs visites de terrain. Vu l'intérêt que ces discussions suscitaient chez les villageoises, il fut décidé d'envoyer plusieurs membres du personnel suivre un stage de formation de 10 jours sur la reconstruction de l'environnement organisé par PEDO en janvier 1988. En tout huit employés de SARTHI participèrent dont les quatre monitrices. Cet événement a marqué un tournant décisif dans la manière dont SARTHI formulait ses priorités. Au lieu d'imposer un plan d'action déterminé à l'avance, pour la première fois, le personnel tenta d'agir en réponse à des besoins exprimés par les femmes elles-mêmes.

L'évolution des groupes de femmes pour aménager les terres incultes doit être vu dans le contexte des événements relatés ci-dessus. En effet, plusieurs membres en provenance des groupes les plus actifs ont participé à l'un ou l'autre des camps, stages de formation ou visites organisés par SARTHI. Ces expériences ont contribué à aider les femmes à surmonter leur timidité et réserves quant au fait de s'engager. 


\section{Aménagement des Terres Incultes Appartenant à des Particuliers}

Les premières tentatives de SARTHI pour remettre en valeur des terres érodées ont eu lieu durant les années de sécheresse de 1985 à 1987. Un nombre important d'animaux sont morts en raison de la pénurie de fourrage et ce qui restait des forêts a été abattu pour être revendu comme combustible afin d'obtenir l'argent nécessaire pour survivre. Créer des emplois à court-terme en incitant les propriétaires à planter des arbres donnant du fourrage et du combustible semblait donc une solution indiquée pour deux raisons. A courtterme cela permettait de générer des revenus et à long-terme d'augmenter la productivité des lopins de terres érodés.

SARTHI demanda donc à ses agents de terrain d'identifier dans leurs régions respectives tous les petits agriculteurs marginaux qui possédaient des lopins de terres incultes et souhaitaient s'associer à cette initiative. Bien que le personnel de terrain féminin était chargé d'encourager les femmes à se joindre au projet, leur participation n'était pas une priorité. A cette époque, les groupes locaux d'hommes ou de femmes ne s'étaient pas encore constitués et ce sont les villageois avec lesquels SARTHI travaillaient déjà sur d'autres projets qui ont servi d'intermédiaire.

Après la saison de plantation, deux ateliers se sont tenus en aout et en novembre 1988 pour permettre aux membres du personnel de faire le bilan. Ils ont révélé que la mise en place du programme s'est heurté à plusieurs obstacles d'ordre socio-politique énumérés ci-après:

- Bien que les propriétaires avaient accepté de participer au programme, la plupart d'entre eux se sont désistés au moment de commencer la préparation du sol, préférant travailler pour des programmes d'assistance à la sécheresse du gouvernement. Cela était dû au fait que SARTHI versait des salaires inférieurs à ceux du gouvernement et parce que SARTHI insistait pour que le travail soit fait suivant des spécifications précises. SARTHI s'est, par conséquent, vu obligé d'embaucher des journaliers pour travailler la terre des propriétaires!

- Initialement, les propriétaires avaient exprimé l'intention de planter des arbres d'espèces variées mais, au moment de passer à l'action, la plupart d'entre eux ne voulaient planter que de l'eucalyptus que l'on croit pourtant responsable d'épuiser le sol et l'eau souterraine. Un homme menaça même d'intenter un procès en dommages et intérêts contre SARTHI si l'organisation plantait autre chose que de l'eucalyptus. (L'eucalyptus est un arbre à croissance rapide abondamment utilisé en Inde comme bois de construction

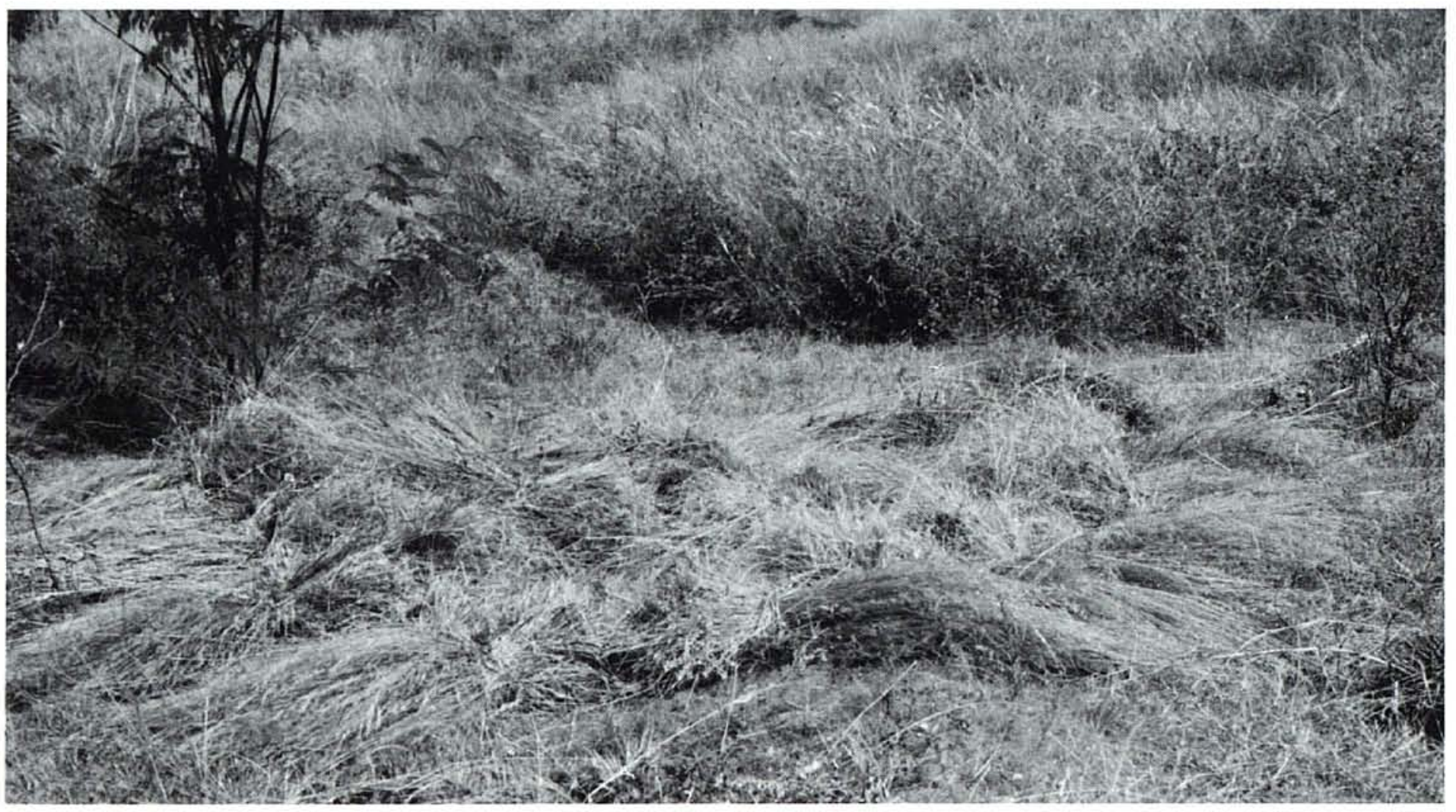




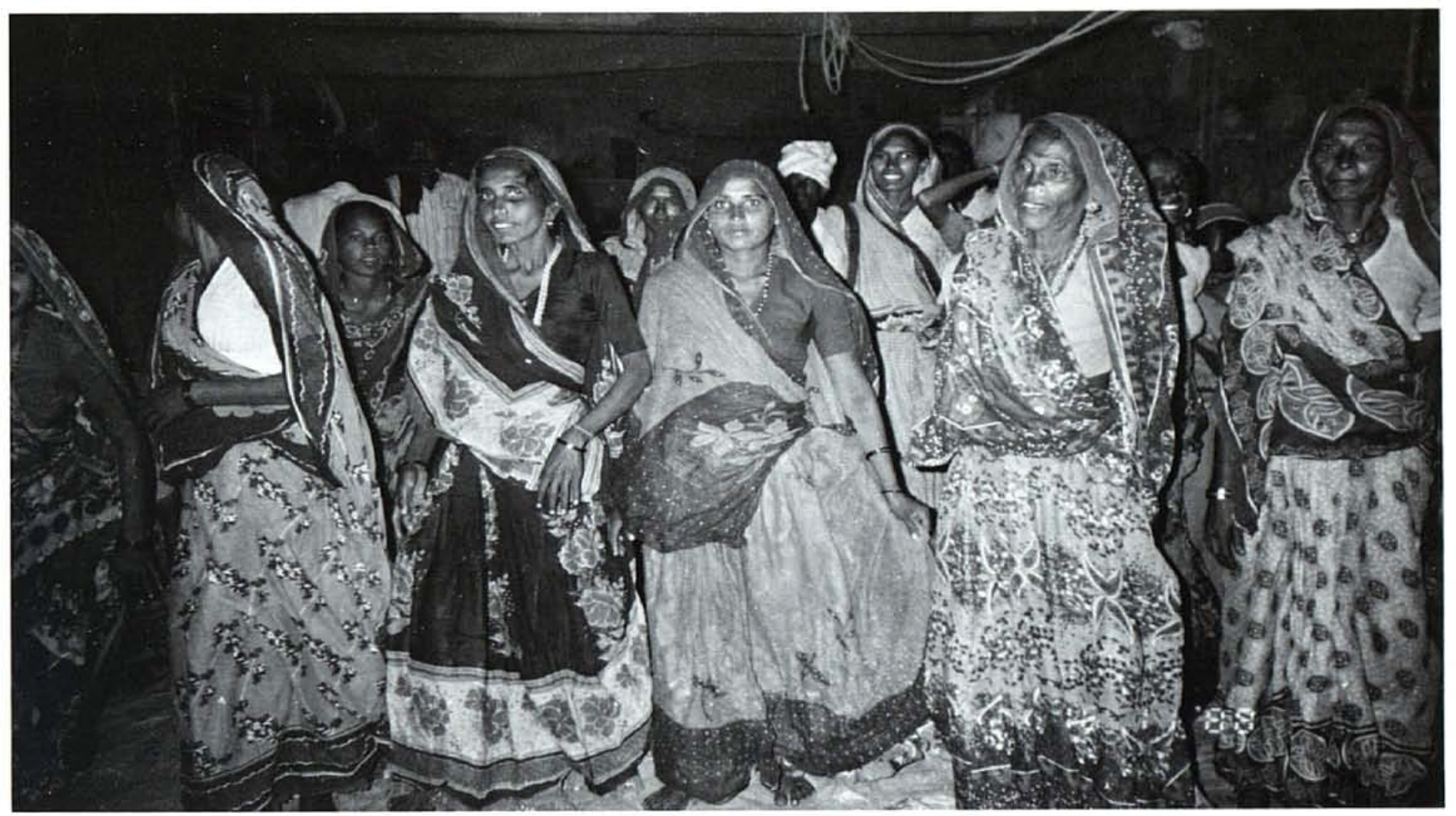

ou pour faire du papier. Jusqu'à ces derniers temps, l'eucalyptus se vendait à un bon prix mais depuis les cours se sont effondrés en raison d'une surproduction).

- Beaucoup de propriétaires étaient hostiles au projet en raison d'une rumeur selon laquelle SARTHI allait éventuellement leur prendre la terre et les arbres.

- Une fois la saison de plantation terminée, peu de propriétaires ont pris la peine de protéger les plants ou de compléter les fossés de délimitation.

Visiblement, ces propriétaires ne voyaient pas les choses de la même façon que SARTHI. Ce qui les intéressait avant tout, c'était de retirer un profit immédiat et non pas de faire un investissement à long-terme.

C'est au cours d'un de ces ateliers que le personnel de SARTHI a entendu parler du travail mené par un groupe de femmes dans le village de Muvasa qui se situe à environ $10 \mathrm{~km}$ des bureaux de SARTHI. Ce programme qui porte sur le développement de quatre hectares de terres communales a été lancé par des employés de SARTHI peu de temps après leur retour du programme de formation de PEDO. Bien que certaines difficultés se sont présentées au moment de son lancement, notamment un conflit avec des habitants du village voisin qui utilisaient ces terre comme pâturages, ce programme connaissait moins de problèmes que les autres.

Ce qui décida SARTHI de travailler avec des groupes pour développer des terres communales, ce fut la réponse que les femmes du groupe de Muvasa apportèrent face à une crise survenue en février 1989. A partir de ce moment. tous les employés de SARTHI se sont mis à organiser des groupes de femmes pour remettre en valeur des terres incultes.

L'incident à l'origine de ce changement est relaté ci-après. Les femmes du groupe de Muvasa venaient de faire la moisson. C'était leur première récolte de foin et elles en étaient fières. Ne sachant pas encore comment ni où l'engranger, il fut décidé de laisser le foin sur le champ.

Un soir, en passant par là, trois hommes saouls du village voisin orit jeté un mégot allumé sur le tas de foin qui s'est enflammé aussitôt. L'incendie a également endommagé les jeunes plants que les femmes avaient pris tant de soin à entretenir.

Les femmes et le personnel de SARTHI étaient ravagés par la nouvelle; un an de travail et d'efforts pour rien! Très vite, pourtant, elles se sont ressaisies et ont décidé d'organiser une réunion pour discuter de ce qu'il fallait faire. Allaient-elles permettre que les trois hommes, qui avaient commis un acte aussi irresponsable, s'en tirent à bon compte? Leur travail n'avait-il donc aucune 
valeur? Si les hommes n'étaient pas punis, tous leurs efforts futurs pourraient être annéantis de la même façon. Les membres du groupe et le personnel de SARTHI se sont ensuite rendus chez les chefs du village ainsi que chez d'autres villageois pour demander leur avis. Presque tous pensaient qu'il fallait punir les coupables. Les femmes ont donc décidé de réclamer des dédommagements aux trois hommes. Au bout de plusieurs discussions, il fut décidé de fixer le montant des dommages à 1400 roupies (USD 78 au taux de change de 1989).

Le groupe convoqua ensuite les hommes et leur fit part de son verdict. Voyant que les femmes n'étaient pas d'humeur à marchander et que le village tout entier étaient derrière elles, les hommes ont accepté de payer les 1400 roupies.

Lorsque l'agent de terrain de SARTHI en poste à Muvasa relata cet incident lors de la réunion du personnel, cela suscita à la fois de l'envie et de l'inspiration chez ses collègues. Qu'on était loin des mesquineries, de l'irresponsabilité et des manipulations dont faisaient preuve les propriétaires. A partir de ce moment là, tous les agents de terrain s'efforcèrent de mettre en place des groupes calqués sur celui de Muvasa dans leurs propres régions.

\section{Naissance du Groupe de Femmes de Muvasa}

Il est utile de donner un apperçu des différentes étapes qui ont mené à la création du premier groupe de Muvasa pour mieux comprendre la dynamique qui opère dans ce groupe et dans tous ceux qui se sont constitués par la suite.

Début 1988, SARTHI avait lancé à Muvasa un programme pour mettre en valeur des terres incultes appartenant à des particuliers. Etant donné que la plupart des propriétaires refusaient de travailler leurs terres, SARTHI a dû recruter des manoeuvres agricoles. La plupart de ces manoeuvres étaient en fait des femmes Adivasi provenant des familles les plus pauvres du village. (En Inde, il est très courant pour un membre d'une tribu de travailler comme journalier).

Tout en travaillant la terre, les femmes parlaient de leur vécu et de leurs problèmes. Elles regrettaient toutes de ne pas être en mesure de participer au programme car les lopins de terre qu'elles possédaient étaient trop petits. Champa Ben et Vikram Bhai, les deux agents de terrain de SARTHI en poste à Muvasa eurent vent de ces propos et se mirent à réfléchir sur la possibilité pour les femmes de s'organiser en groupe et de développer un lopin de terre communale. Cette idée fut accueillie avec beaucoup d'enthousiasme chez les femmes et SARTHI accepta de prendre en charge les frais de préparation du sol et de plantation. (La plupart des villages en Inde possèdent des pâturages communaux qui sont accessibles à tous les villageois. La gestion de ces terres tombe sous la responsabilité des panchayats. Nombre de villages disposent également de "terres incultes fiscales", c'est-à-dire appartenant au Fisc mais se trouvant dans la limite de leur panchayat. Utilisées au même titre que des terres communales par les villageois, le Fisc a pourtant le pouvoir de transférer ces terres à un individu ou un groupe).

Des réunions improvisées sur les différents lieux de travail ont permis d'établir la manière dont le groupe allait fonctionner. Toutes celles qui voudront se joindre au groupe devront participer à la préparation du sol et à la plantation. La protection de la plantation sera assurée collectivement par le groupe. La récolte sera partagée équitablement entre tous les membres du groupe. Toutes les décisions relatives à la plantation seront prises collectivement. Seules les femmes auront le droit de s'affilier au groupe puisque ce sont elles qui souffrent le plus lorsqu'il y a pénurie de combustible et de fourrage. Toutes les femmes intéressées du village, y compris les femmes Patel appartenant aux castes supérieures, seront invitées à se joindre au groupe. II fut décidé après réflexion de ne pas inclure les femmes Patel plus aisées puisqu'elles ne sont pas autorisées à travailler en dehors du foyer et que, de toute façon, elles ont déjà trop de travail vu que leurs familles possèdent des fermes plus grandes.

Lorsque le groupe commença à se former, il fut décidé d'approcher le panchayat. Le panchayat leur remis un "certificat de non objection". Ce certificat les autorisait à planter quatre hectares de terre communale avec la garantie de renouveler le bail après 10 ans et leur permettait de garder l'intégralité de la récolte.

Les femmes les plus dynamiques ont ensuite fait du porte-à-porte pour demander aux autres femmes du village à l'exception des Patel si elles voulaient se joindre au groupe. En tout, 29 femmes sont devenues membres. Elles provenaient toutes des familles les plus pauvres du village et certaines possédaient moins d'un are de terre. Toutes étaient mariées mais d'âge différent. 
Surmonter le Problème de la Méfiance Chez les Hommes

Même avant que le groupe ne commence à travailler la terre, les hommes du village se sont interrogés sur la raison pour laquelle l'organisation travaillait seulement avec des femmes. Les maris de certains membres considéraient que les réunions étaient une perte de temps et que le temps serait mieux passé à faire le ménage. Au niveau de la famille, chaque femme a dû faire preuve à la fois de fermeté et de tact. Elles commençèrent à se lever plus tôt pour finir le ménage avant d'aller travailler la terre ou d'assister à des réunions.

Pour appaiser la méfiance des hommes, SARTHI a convié tous les villageois à une réunion afin de leur expliquer pourquoi l'organisation travaillait avec un groupe de femmes. La discussion porta sur la pénurie de combustible et de fourrage, l'impact que cela avait sur la vie des femmes et le fait que ces dernières avaient un intérêt tout particulier à remédier au problème. Bien que ce débat ainsi que d'autre organisés par la suite ont contribué à calmer les esprits, de nombreux hommes continuèrent à propager des rumeurs que SARTHI allait enlever les femmes, voler leurs bijoux, etc. Et certains hommes allèrent même jusqu'à épier les femmes lorsqu'elles se réunissaient pour écouter leurs conversations.

Mais petit à petit, comme le travail du groupe progressait à un rythme régulier et que les femmes devenaient plus sûres d'elles, les rumeurs et les appréhensions ont cessé. SARTHI a également aidé la situation en sollicitant plusieurs hommes du village à participer à certains de ses projets pour leur permettre de se familiariser avec l'organisation.

\section{Création d'un Espace Social Légitime pour les Femmes}

En faisant en sorte qu'il soit socialement acceptable pour les femmes de s'organiser pour faire face à un de leurs problèmes les plus cruciaux, il fut possible de créer pour la première fois un espace légitime pour les femmes afin de se rencontrer régulièrement. Et pour la première fois, un groupe de villageoises a obtenu le droit d'exploiter un lopin de terre communale en fonction de leurs besoins et priorités. Bien que ce lopin de terre ne leur appartient pas, personne ne leur conteste le droit de l'exploiter collectivement. Cela constitue en soi une percée considérable.
SARTHI a pris soin de s'assurer que des représentantes de chaque groupe étaient présentes aux négociations avec les panchayat (composés presque exclusivement d'hommes) et avec le fonctionnaire chargé de tenir à jour les titres de propriété pour obtenir les documents nécessaires pour louer la terre. Au cours de ce processus, les femmes ont pu se familiariser avec les questions relatives à la terre. Elles avaient toujours cru que seuls les hommes étaient capables de s'occuper de telles questions. Elles voyaient bien que ces croyances n'étaient qu'un mythe.

SARTHI a également laissé aux femmes le soin de décider ce qu'il fallait planter et comment organiser le travail. Par exemple, au lieu de venir travailler à des heures fixes, il fut convenu de mettre en place un horaire souple, de noter la quantité de travail accomplie par chaque membre et de payer en fonction du rendement. Ce système a permis aux femmes d'allier le travail à la plantation aux corvées ménagères. Petit à petit, comme les femmes devenaient plus sûres d'elles, elles ont exigé de leurs maris qu'ils s'occupent des corvées domestiques lorsqu'elles partent travailler, assistent à des réunions ou participent à des camps de formation.

\section{Tour d'Horizon de l'Expérience des Différents Groupes}

A I'heure actuelle, SARTHI travaille avec 17 groupes de femmes qui sont éparpillés dans les cinq régions où SARTHI est implanté. Tous ces groupes travaillent soit sur des panchayat gauchor (des pâturages communaux) soit sur des terres appartenant au Fisc. Le processus de mise en place des groupes de femmes dans d'autres villages a quelque peu différé de celui du groupe de Muvasa. Dans certains cas, l'initiative est venue de SARTHI qui, après avoir trouvé un lopin de terre disponible, a essayé de convaincre les femmes locales de s'organiser en vue de le développer. Dans d'autres, ce sont les chefs de certains villages qui, inspiré par le groupe de Muvasa, ont approché SARTHI avec la demande d'établir un groupe similaire dans leur village pour développer un lopin de terre qu'ils avaient réservé à cette fin.

Comme l'on peut s'y attendre, chaque groupe a connu une expérience quelque peu différente. Alors que certains fonctionnent bien, d'autres connaissent des problèmes et d'autres encore à peine constitués se sont dissouds. Le 
développement de chaque groupe dépend d'une variété de facteurs complexes, notamment:

- les rapports de force à l'intérieur du village et l'existence de conflits au sujet de l'utilisation des terres communales;

- le statut des femmes et l'étendue de leur ouverture au monde extérieur;

- l'attitude des hommes envers les femmes prenant de telles initiatives;

- la compétence, l'expérience et les capacités de leadership des agents de terrain, notamment du personnel féminin; et

- l'étendue de la pénurie de combustible et de fourrage dans la région.

Une des stratégies les plus efficaces pour consolider les groupes qui manquent de cohésion, c'est de les mettre en rapport avec des groupes qui existent depuis longtemps et qui fonctionnent bien.

\section{Interaction entre les Groupes}

Tous les groupes qui fonctionnent bien à présent ont connu des problèmes mais c'est la détermination et les méthodes assurées dont ils ont fait preuve pour surmonter ces obstacles qui ont contribué à consolider les groupes et à asseoir leur crédibilité. Par ailleurs, la nécessité pour ces groupes de prendre de telles mesures dès le départ a eu pour effet de minimiser les problèmes qui se sont présentés par la suite.

En plus des rencontres entre les membres des groupes nouvellement crées et des groupes plus anciens, SARTHI vient tout récemment d'organiser un atelier de deux jours pour permettre aux représentantes de plusieurs groupes d'échanger leur expérience associative. L'une des plus grandes difficultés auxquelles se heurtent nombre de nouveaux groupes c'est la tentative par des chefs de village manipulateurs d'utiliser les femmes pour avancer leurs propres intérêts. C'est exactement ce qui est arrivé au groupe du village de Paderi comme en témoigne le récit ci-après.

Le groupe de femmes de Paderi fut établi en 1990 et SARTHI aida le groupe à planter des arbres d'espèces variées sur un lopin de terre érodé. Au début, le groupe fonctionnait bien et apparaissait uni. Les hommes du village se tenaient à l'écart de l'endroit où le groupe se réunissait de crainte que les femmes ne leur demandent de servir de l'eau, une tâche tradition- nellement réservée aux femmes. Peu à peu, pourtant, l'unité du groupe commença à se dissoudre au point de laisser la plantation sans protection.

Les quatre membres du groupe qui participaient à l'atelier affirmèrent que c'était la faute d'un homme saoul qui avait refusé de coopérer avec le groupe et avait menacé d'attaquer tous ceux ou celles qui l'empêcherait de faire paitre ses animaux sur la plantation. Les femmes s'étaient rendues à l'atelier pour obtenir de SARTHI l'argent nécessaire pour embaucher un gardien à plein temps. SARTHI refusa d'octroyer l'argent estimant que le groupe était tout à fait capable d'assurer lui-même la protection de la plantation. Mais les femmes ne voulaient rien entendre et persistèrent dans leur demande.

Des discussions informelles avec les agents de terrain locaux ont révélés en fait que les maris de deux des membres du groupe était candidat au poste de sarpanch (le dirigeant du panchayat élu). Tous les deux essayaient de se servir de leurs femmes pour extraire de SARTHI un bénéfice qui renforcerait leur propre position à l'intérieur du village. Les femmes s'étaient laissées prendre à ce jeu ce qui a eu pour effet de diviser le groupe en deux camps opposés.

On demanda aux représentantes des autres groupes qui étaient présentes si elles avaient rencontré des problèmes similaires et ce qu'elles feraient si elles étaient confrontées à la même situation que les femmes du groupe de Paderi.

Karsan Ben du groupe de Wandki prit la parole pour parler d'un incident qui s'était produit plusieurs mois auparavant. En se rendant sur leur plantation, le groupe eut la surprise de découvrir quatre ou cinq chèvres, qui appartenaient au sarpanch de leur village, en train de brouter. Dix huit femmes se sont aussitôt rassemblées pour attraper les animaux et les enfermer dans l'enclos appartenant au panchayat. Ce qui est très intéressant, c'est que cet enclos est maintenu par nul autre que le sarpanch. II n'a pourtant eu le droit de reprendre ses chèvres qu'après avoir payé une amende de 700 roupies au groupe. Les autres villageois voyant que même le sarpanch n'a pas été épargné ont bien compris qu'il valait mieux se tenir à l'écart.

Avant cela, au moment de commencer à planter leur terre, les membres du groupe de Wandki avaient décidé d'autoriser les hommes de leur famille à venir travailler sur leur plantation. Très vite, les femmes se rendirent compte que les hommes n'étaient pas très consciencieux et que leur travail laissait beaucoup à désirer. Par exem- 


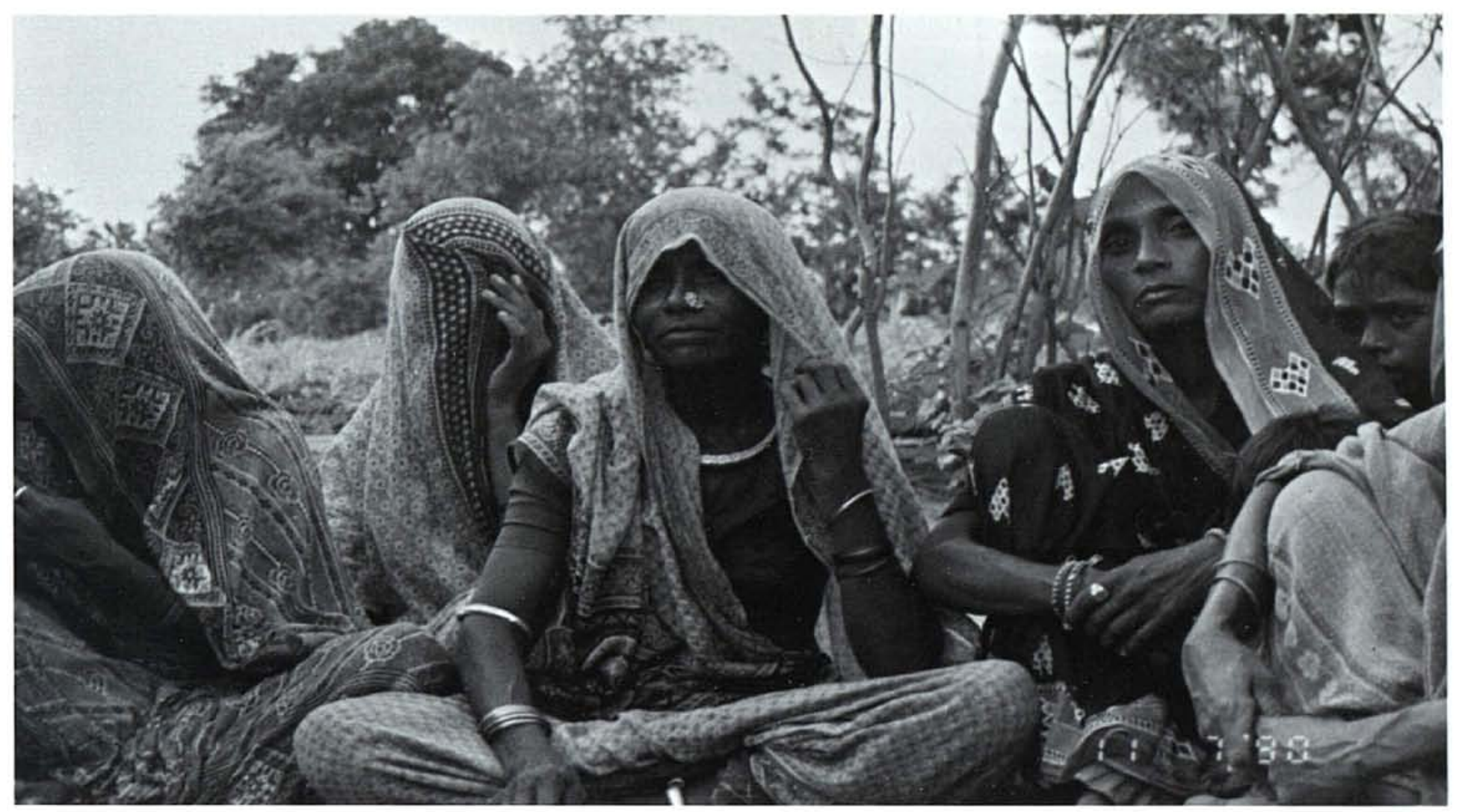

ple, en ramenant les plants de la pépinière, les hommes systématiquement en jetaient une partie le long de la route pour alléger leur chargement. Très mécontentes de ce gâchis, les femmes ont formellement interdit aux hommes, y compris aux maris, de mettre les pieds sur leur plantation à l'avenir.

De même, le groupe a appris à rester ferme sur sa décision de ne pas permettre aux hommes d'assister à leur réunion. Comme les femmes de la région pratiquent le purdah (le fait de se couvrir la tête et le visage) lorsqu'elles se trouvent en présence d'hommes plus âgés appartenant à leur famille, elles ne peuvent pas parler ouvertement devant les hommes ce qui va à l'encontre de l'objectif que les femmes se sont fixés en se réunissant séparément. Une conversation entendue par hasard entre des hommes du village révèle jusqu'à quel point les femmes ont en fait réussi à faire de leur réunion une "affaire de femmes". Ils se racontaient qu'il valait mieux s'abstenir d'aller aux réunions des femmes sinon elles exigeraient d'assister aux réunions ouvertes exclusivement aux hommes!

Ce fut ensuite au tour de Kapuri Ben du groupe de Wandariya de parler d'une expérience que son groupe avait connu au tout début. Après avoir fini de travailler leur terre, les femmes décidèrent de planter une haie d'ipomea (un arbrisseau non comestible par le bétail) tout autour du champ. Un homme qui habitait à proximité du champ a déraciné tous les Ipomea car il voulait continuer de faire paitre son bétail sur cette terre. Avec l'aide de SARTHI, le groupe a déposé une plainte auprès de la police et l'homme a dû payer une amende élevée. Après cela, il ne s'est plus jamais aventuré à faire paitre son bétail sur la plantation. Toutefois, pour compenser l'homme de la perte du pâturage et pour éviter le risque de s'aliéner ses sympathies, le groupe l'a engagé comme gardien.

Kapuri Ben déclara qu'aucun membre du groupe n'avait jamais été menacé ou battu par un homme. Et que s'ils essayaient, ils se feraient battre à leur tour par les femmes!

Nani Ben du groupe de Musava remarqua que si le groupe de Paderi était uni, aucun ivrogne n'oserait menacer les membres du groupe ou faire paitre son bétail sur leur plantation. Elle parla ensuite de la situation face à laquelle son groupe était confronté récemment. Comme la plantation du groupe se situe assez loin de l'endroit où habite les femmes, un homme vivant à proximité en profita pour laisser paitre son bétail dans leur champ durant la nuit. II coupa même du fourrage en douce. Les femmes décidèrent de prendre l'homme la main dans le sac.

Un jour, 15 membres du groupe se cachèrent dans la plantation. Lorsque l'homme arriva avec son bétail, les femmes sortirent de leur 
cachette et confrontèrent l'homme. Celui-ci se mit à lancer des insultes et affirma que rien ne pourra l'arrêter. C'est alors que Kanta Ben, l'une des femmes du groupe, le prit par la peau du cou et exigea de lui qu'il présentent des excuses pour son comportement grossier. L'homme pris de panique s'excusa. Depuis lors, il n'a jamais interferé avec la plantation.

Des membres du groupe de Gara voulaient savoir quelle était l'utilité de former un groupe si tellement de femmes allaient continuer à se laisser intimider par un ivrogne. La seule solution se trouvait dans l'action collective.

Les femmes de Paderi finalement réalisèrent que la solution à leur problème n'était pas d'embaucher un gardien et d'insister pour que SARTHI leur donne l'argent nécessaire. Elles firent la promesse que dès leur retour, elles essaieraient de reprendre leur groupe en main.

\section{Rendements des Plantations}

Les groupes plus anciens ont déjà obtenu plusieurs récoltes de biomasse (fourrage vert, semences, quelques légumes et un peu de combustible) et sont en train d'affiner leur système de moisson et de distribution. Le groupe de Muvasa a déjà récolté du fourrage trois fois. La première récolte a été brûlée mais au cours de la deuxième année, les femmes ont décidé de garder la moitié de la récolte et de vendre l'autre moitié. La troisième année, les femmes ont gardé l'intégralité de la récolte qu'elles se sont partagées et ont ramenée chez elles pour nourrir leur bétail.

Les plantations des groupes de Wandariya et de Wandki n'ont pas donné de bonnes récoltes de fourrage vert puisque le sol est très caillouteux. Par contre, le groupe de Wandariya a déjà produit du fourrage de feuilles d'arbres et du combustible grâce à ses efforts de régénération d'arbres existants. La régénération se pratique en coupant les multiples tiges émises par le rhizome existant pour ne permettre qu'à une seule tige saine de pousser. Les produits de la coupe sont utilisés comme combustible.

Le groupe de Gara a l'intention d'autoriser ses membres à couper du fourrage vert cette année puisqu'il s'agit d'un fourrage de plus forte valeur nutritive. En échange, chaque membre sera tenu de verser une somme minime, ceci afin de renflouer la caisse commune. En remplaçant graduellement la variété herbacée locale par un fourrage de meilleure qualité, toutes les plantations seront bientôt en mesure de produire un fourrage à plus forte valeur nutritionnelle. Les groupes se sont également mis à produire des semences destinées à être utilisées sur de nouvelles plantations.

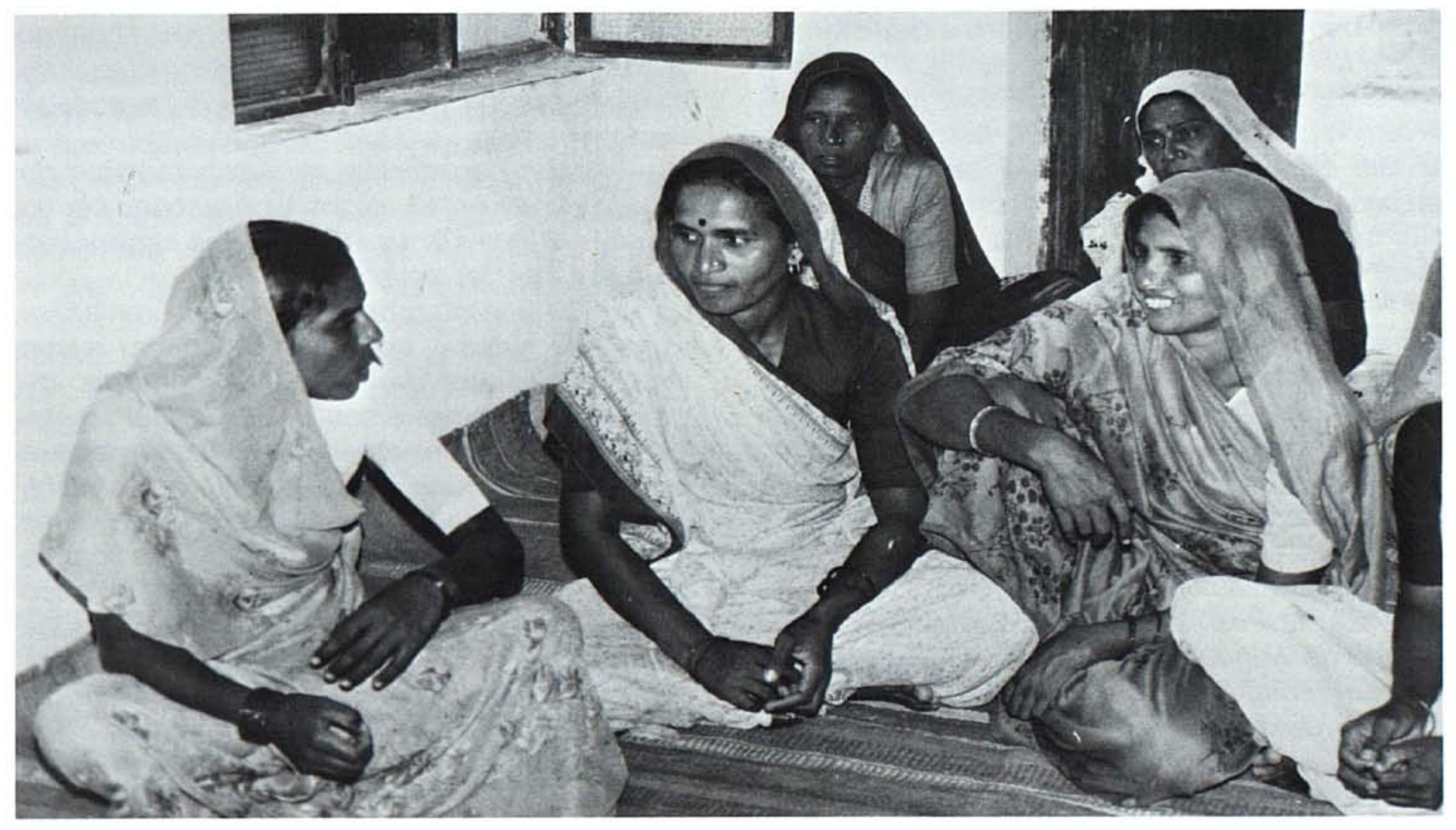




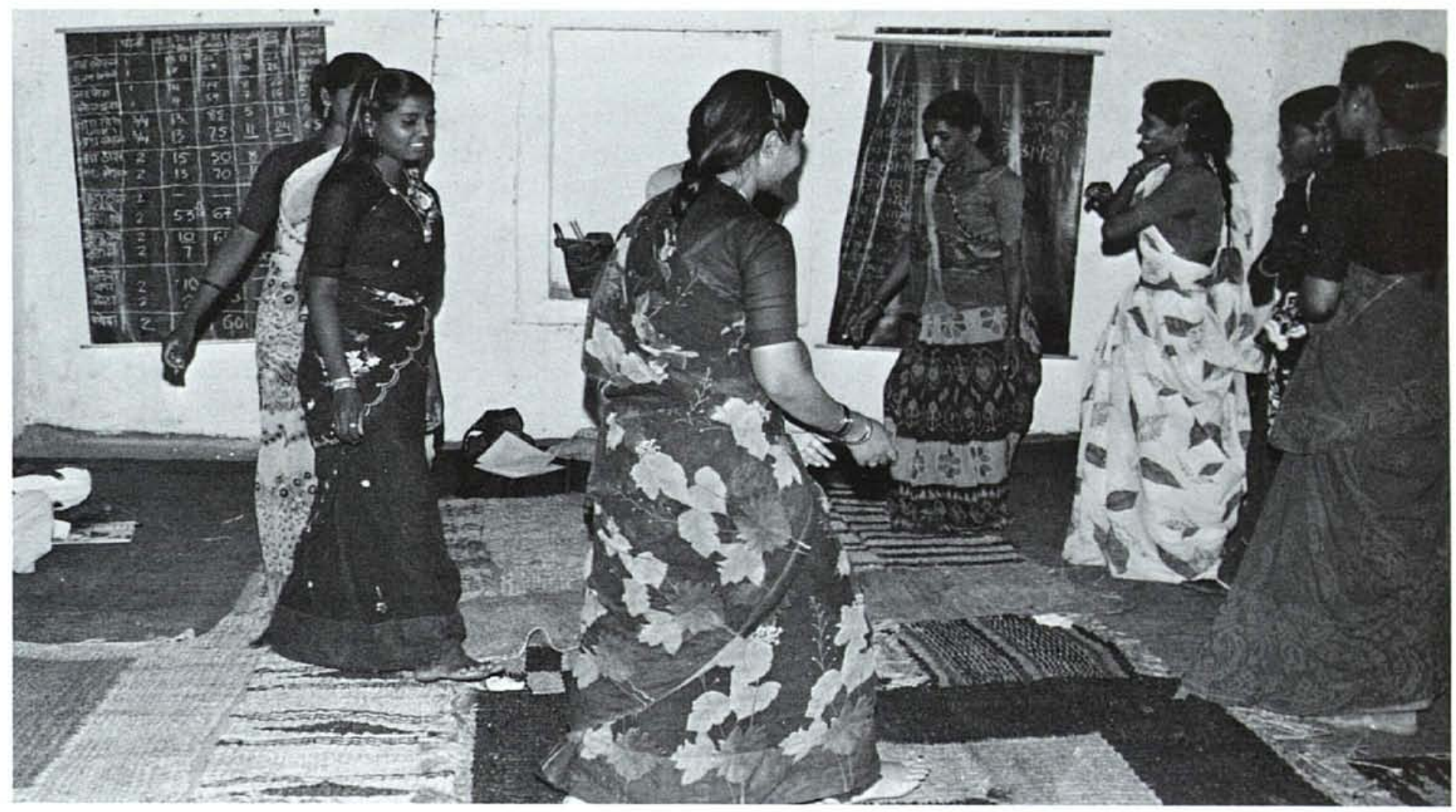

\section{Autres Bénéfices}

En plus de la production de biomasse, la participation aux activités de groupe a contribué à améliorer d'autres aspects de la vie des femmes. Par exemple, les visites de suivi des agents de santé ont révélé que les villageois souffraient de carences alimentaires aigues (particulièrement en vitamine $\mathrm{A}$ ce qui peut engendrer la cécité chez les enfants et les femmes enceintes). Des discussions sur le mode d'alimentation, les croyances et les pratiques alimentaires ont révélé que l'alimentation n'était pas suffisamment variée et que la consommation de lait, de produits laitiers, de légumes-feuilles, de fruits et, dans une certaine mesure, de légumes en général, était insuffisante. Les produits laitiers et les fruits sont difficiles à trouver dans la région de même que les légumes-feuilles durant les mois d'été secs. Mais même lorsque ces aliments sont disponibles, les femmes n'ont souvent pas le temps de les préparer en raison de leur charge de travail excessive.

Ces discussions ont à leur tour conduit SARTHI à intégrer dans ses programmes d'aménagement des terres incultes des variétés d'arbres à forte valeur nutritionnelle. Parmi ces variétés figurent l'aonla (phyllanthus emblica), un arbre local dont le fruit est très riche en vitamine $C$, et le sengwa (moringua oleifera), dont les feuilles sont riches en vitamines $A$ et $C$. Ces feuilles peu- vent être cuites légèrement et consommées durant les mois d'été lorsque d'autres légumesfeuilles ne sont pas disponibles.

En 1989, une équipe de SARTHI se mit à rassembler des informations sur les connaissances des habitants au sujet des arbres et arbustes locaux afin d'identifier les espèces à intégrer aux programmes d'aménagement des terres incultes. Cette enquête indiqua clairement que I'utilisation d'herbes et de plantes médicinales pour soigner les problèmes de santé des femmes était une pratique traditionnelle très courante. En effet, les forêts locales abondent en plantes et en herbes médicinales. La décision de SARTHI d'incorporer des espèces ayant des vertus médicinales devint donc une nouvelle facette de son programme.

En 1989-1990, suite aux groupes de remise en valeur des terres incultes, des femmes de différents villages ont formé une vingtaine de groupes d'épargne. Ces groupes ont émergés à la suite d'un besoin grandement ressenti par les femmes d'avoir accès à leur propre source de crédit en cas de coup dur ce qui leur évite d'avoir à emprunter de l'argent aux usuriers locaux qui demandent des taux d'intérêts exhorbitants.

Les réunions intergroupes sont devenues le forum pour les constructrices de fourneau, les agents de santé et les paravets de dialoguer avec les villageoises pour les sensibiliser à divers pro- 
blèmes et augmenter leurs connaissances. En outre, plusieurs camps se sont tenus dans les bureaux de SARTHI en faveur de villageoises préalablement sélectionnées pour leur apprendre à mobiliser et à motiver. Finalement, plusieurs camps portant sur l'eau potable et l'environnement ont été organisés dans plusieurs villages. Nombre de femmes ont eu l'occasion d'assister à des melas, ateliers et camps ${ }^{2}$ organisés par d'autres organisations et portant sur des thèmes revêtant un intérêt tout particulier pour elles. Des échanges entre les membres de différents groupes locaux ont également eu lieu.

La possibilité pour une femme de quitter son village pour rencontrer et parler avec de nombreuses autres femmes est en soi une expérience très enrichissante. En effet, les femmes n'ont que peu d'occasions de dialoguer avec d'autres femmes en dehors de leurs réseaux familial et communautaire. Même à l'intérieur de la communauté, les seules fois où les femmes se rencontrent c'est pour assister à des marriages, des enterrements ou des cérémonies religieuses où elles sont confinées dans leur rôle traditionnel. Grâce au programme de SARTHI, les femmes ont la possibilité de partager leur vécu avec des femmes appartenant à d'autres castes et en provenance d'autres communautés. En plus d'acquérir des connaissances nouvelles, elles ont la possibilité de surmonter quelque peu leurs sharam $^{3}$, timidités et inhibitions traditionnelles.

A présent, les activités en faveur des femmes sont devenues la composante principale du programme de SARTHI. En raison de la réponse positive des femmes à chaque effort de sensibilisation, SARTHI a essentiellement remplacé ses projets orientés vers des bénéficiaires individuels par des projets axés sur l'organisation et la mobilisation de femmes et de jeunes ainsi

\footnotetext{
2 Les camps qui durent de un à cinq jours sont des événements au cours desquels un groupe ou un village spécifique obtient des informations sur un ou plusieurs sujets choisis. Les melas sont similaires aux camps mais le nombre de participants est plus important. Les melas et les camps sont parfois organisés dans des centres de formation ce qui oblige les participants à passer la nuit loin du foyer. Les frais de voyage et autres coûts sont normalement pris en charge par l'organisateur, en l'occurence le gouvernement ou une ONG.
}

\footnotetext{
3 II n'y a pas de traduction adéquate du terme indien sharam qui signifie un sens aigu de honte et d'embarras sur le fait d'avoir à agir ou à se comporter en dehors de son rôle traditionnel. C'est cette notion de sharam. plus que toute autre, qui fait que les femmes indiennes restent si fortement attachées à la tradition.
}

que d'hommes. Parce que la hiérarchie interne dans les familles et les communautés peut constituer un véritable handicap à la participation de certains acteurs comme les femmes ou les jeunes à la vie économique et sociale, le recours à la dynamique de groupe peut être un moyen efficace pour surmonter ce handicap.

\section{Directions Futures}

Bien que tous les groupes pourront bientôt récolter de la biomasse, un problème majeur auquel ils sont confrontés c'est que, dans la plupart des cas, la superficie de leur plantation est trop petite pour satisfaire tous leurs besoins et d'autres terres communales ne sont malheureusement pas disponibles. Si les groupes de femmes essayaient d'aggrandir la superficie de leur plantation, ils risqueraient d'entrer en conflit avec des familles qui dépendent de ces terres pour faire paitre leur bétail.

Les femmes, à présent, essaient de voir comment d'autres terres de la région appartenant au secteur public sont gérées. Parmi celles-ci, la catégorie qui semble offrir le plus de potentiel pour améliorer la productivité est la forêt qui est contrôlée par le Département Forestier de l'Etat. Fort heureusement, le Département Forestier de Gujarat réalise qu'il n'est pas possible d'améliorer la gestion des forêts sans la coopération des villageois qui habitent aux alentours. C'est pourquoi, un plan de co-gestion est en cours d'élaboration et SARTHI a commencé à explorer la possibilité pour les groupes de femmes de s'associer à cette initiative.

La question de la reconnaissance du droit des femmes d'exploiter la terre est un autre point qui mérite de l'attention. Tous les groupes ont reçu de leurs panchayats des "certificats de nonobjection" ainsi que des résolutions leur donnant l'autorisation d'exploiter la terre et de garder les produits de la terre pendant plusieurs années. Pourtant la validité légale des ces certificats et résolutions reste ambigue. La question de savoir si un panchayat nouvellement élu a le droit d'annuler ou de retirer un certificat délivré par le gouvernement précédent soulève le plus d'inquiétude.

II est possible toutefois de contourner de telles ambiguités légales en donnant aux femmes le pouvoir de revendiquer leurs droits; le panchayat nouvellement élu n'aurait pas le courage de reprendre la terre par peur des représailles. Pourtant, ceci n'est possible qu'en élargissant la base du soutien aux groupes parmi les autres mem- 


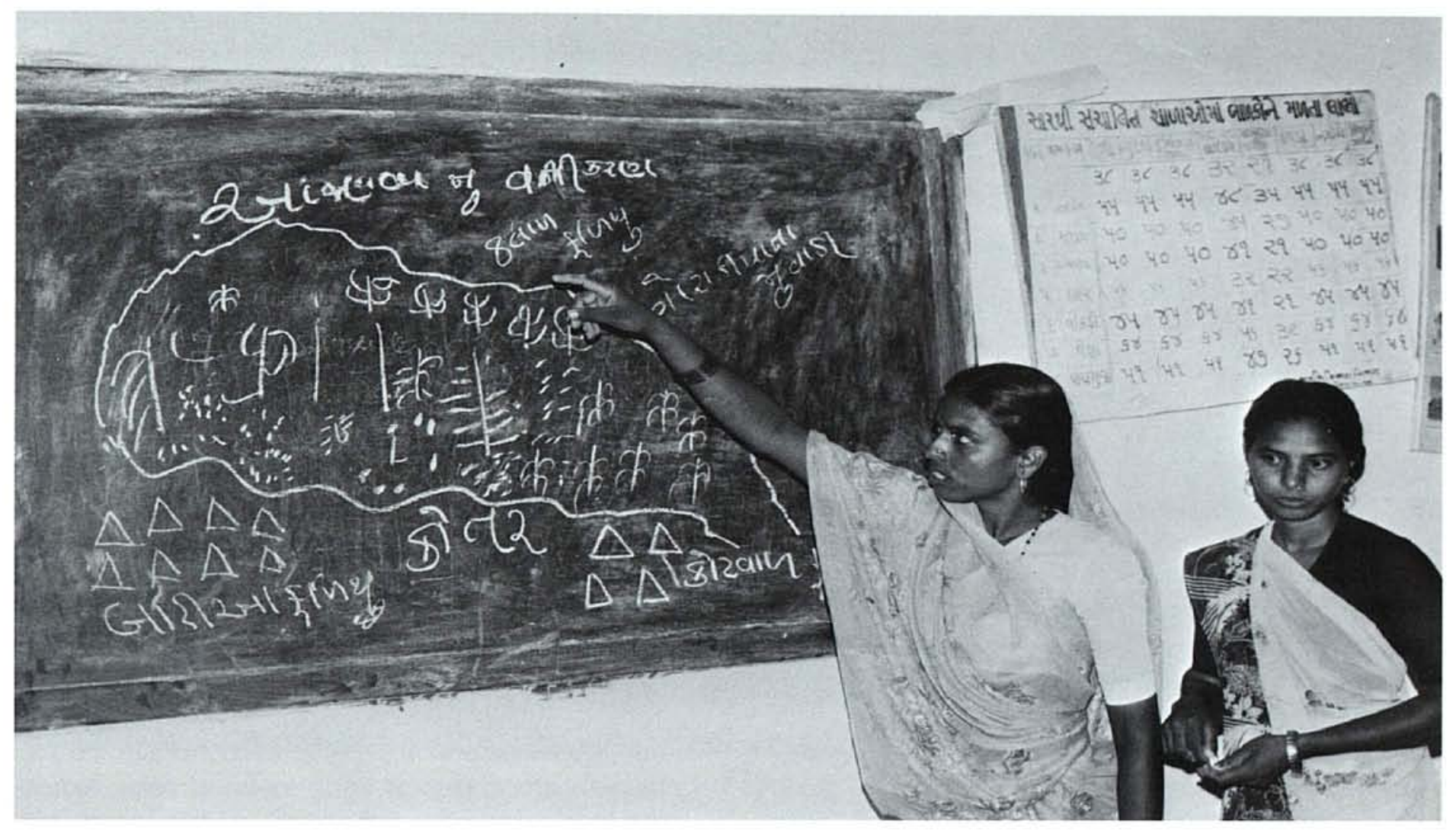

bres de la communauté et en renforçant le poids politique des femmes à l'intérieur des institutions locales. Jusqu'à présent, la plupart des groupes n'ont eu aucun problème à obtenir des membres de leurs panchayats qu'ils les soutiennent en situation de crise ou de conflit en invoquant qu'il incombait au panchayat d'honorer les termes de leurs certificats.

La survenue de conflits potentiels entre plusieurs groupes se disputant âprement les maigres ressources en provenance des terres communales reste très élevée. II ne fait aucun doute que la détermination des femmes à revendiquer leurs droits sera soumise à de nombreux tests. Par exemple, le groupe de Muvasa est, à présent, engagé dans une autre bataille. Apparemment, six familles du village voisin ont abattu et volé la plupart des arbres qui se trouvaient sur leur plantation. Le groupe a déposé une plainte auprès de la police et a demandé que les panchayats des deux villages prennent des mesures pour punir les coupables. C'est la première fois que deux panchayats sont impliqués ce qui a eu pour effet de créer des tensions entre les villages. SARTHI a aidé les femmes à organiser une manifestation pour porter leur cas à l'attention d'autres villages et élargir la base de leur soutien. A l'heure qu'il est, il n'est pas possible de savoir comment ce conflit va se terminer. Mais étant donné la détermi- nation dont les elles ont fait preuve par le passé, il est certain qu'elles continueront à se battre. Et la bataille promet d'être longue.

\section{Lessons à Retenir}

Etant donné que ce sont essentiellement les femmes qui amassent et utilisent la biomasse, ce sont elles qui sont par conséquent le mieux placé pour participer à des projets de remise en valeur de terres incultes puisqu'elles possèdent à la fois le savoir-faire et la motivation nécessaires. Mais étant donné que la société est sous la domination des hommes, il est essentiel d'aider les femmes à surmonter un certain nombre d'obstacles qui les ont traditionnellement empêcher de travailler ensemble, de s'organiser et de se faire entendre au sein de la communauté. Les efforts de SARTHI pour mobiliser les femmes autour d'activités d'aménagement de terres incultes permettent de tirer des leçons importantes dans deux domaines: la gestion des ressources naturelles et l'émancipation des femmes.

1. Aider les femmes à s'organiser en groupe leur donne le pouvoir dont elles ont besoin pour briser le carcan familial et communautaire. Les besoins collectifs des femmes ne pourront être satisfaits qu'à condition de modifier la division du travail à l'intérieur du foyer et plus globalement au sein de la société toute entière. La participation 


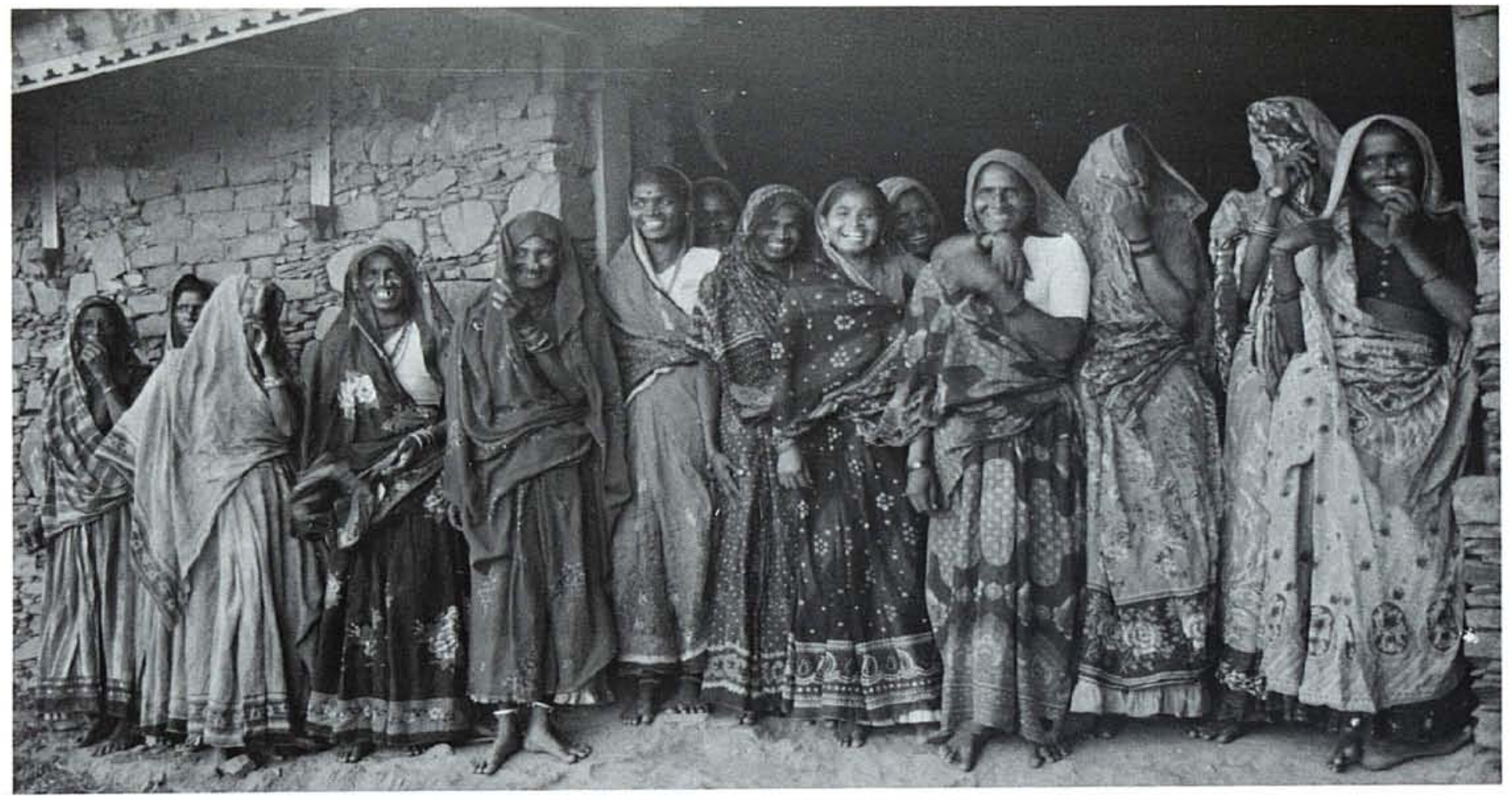

des femmes aux activités de remise en valeur des terres incultes leur a permis de défier la hiérarchie familiale en obtenant, par exemple, que les maris s'occupent des corvées ménagères lorsqu'elles assistent à des réunions ou participent à des camps de formation. Elles ont aussi appris à tenir tête à leurs maris lorsqu'il s'agit d'honorer des décisions prises par le groupe. Et finalement, plusieurs femmes ont été en mesure de mettre un terme à la violence domestique dans leur foyer grâce au soutien du groupe.

2. L'un des points forts des différents groupes de femmes est qu'ils opèrent selon des principes démocratiques. Les décisions sur la quantité, le rendement et la qualité du travail sont prises en commun et tous les participants se partagent équitablement le travail et la récolte. Ce modèle contraste nettement avec celui qui prévaut dans les institutions sociales, politiques et gouvernementales contrôlées par les hommes qui ont tendance à privilégier les intérêts de groupes spécifiques comme ce fut le cas avec les propriétaires avec lesquels SARTHI a travaillé initialement.

3. En établissant dès le départ les modalités de fonctionnement et de participation, le groupe de Muvasa a crée des attentes réalistes sur les droits et les responsabilités des membres. Par exemple, pour faire partie du groupe, les membres étaient tenus de travailler la terre ce qui leur donnait droit à une partie de la récolte. Ceci a naturellement conduit à l'exclusion des femmes appartenant aux castes supérieures puisqu'elles n'auraient pas pu trouver le temps de travailler sur la plantation. Dans le même temps, ce processus a permis d'assurer la cohésion du groupe étant donné que les femmes venaient toutes du même milieu.

4. Pour faciliter la création d'un espace pour les femmes au sein d'une communauté, il faut des agents de terrain spécialement formés à cet effet. C'est une condition minimale si on veut organiser les femmes, particulièrement dans les endroits où elles n'ont pas la possibilité de se rencontrer en dehors de leur réseau familial. Lorsque un tel espace est crée, les femmes peuvent se faire entendre et obtenir l'approbation implicite de leur communauté.

5. En donnant aux femmes l'occasion de se déplacer en dehors de leur foyer et de leur communauté, il est possible de renforcer leur sens de la puissance collective. Loin d'être purement symbolique, la mobilité géographique donne également aux femmes de différents milieux l'occasion de recevoir une formation, d'acquérir des compétences et de se familiariser avec les structures et le fonctionnement d'institutions gouvernementales. La participation à des réunions "extérieures" est une excellente occasion pour les femmes d'acquérir plus de confiance en soi sur le fait d'avoir à prendre la parole devant un grand nombre de personnes. 
6. Au lieu de se contenter d'imposer un programme déterminé à l'avance, il vaut mieux sensibiliser les agents de terrain aux préoccupations des femmes et les encourager à développer des stratégies appropriées localement en vue d'assurer leur émancipation. En effet, une telle approche aboutit à la mise en place de projets qui ont le plus de chances de réussir. Le processus de formation des groupes pour remettre en valeur les terres incultes sert ainsi de modèle aux autres activités de développement de SARTHI.

7. En insistant pour que leurs panchayats respectifs interviennent en leur faveur lorsque leur droit d'exploiter la terre fut remis en question à l'intérieur comme à l'extérieur de leurs communautés, les groupes ont réussi à faire passer le message que leurs acquis étaient valides. En exigeant que les panchayats fassent respecter les termes des certificats qu'ils avaient délivrés, les femmes ont établis qu'elles étaient des citoyennes à part entière ayant les mêmes droits que les autres. Avaient-elles opté pour une stratégie moins visible, elles auraient sans aucun doute marginalisé leur position. II est fort probable que cette action poussera les femmes à voter lors des prochaines élections régionales pour promouvoir leurs intérêts, augmentant de ce fait la participation des femmes au processus politique.

8. Les groupes de femmes ont évolué à la fois en récompensant la productivité et en défiant directement et immédiatement tous les efforts visant à minimiser ou à exploiter leur travail. Elles ont réussi à obtenir des dédommagements pour les dégats causés à leur plantation, ont puni ou confronté tous ceux qui ont essayé de les empêcher d'accomplir leur travail. II ne fait aucun doute que la rapidité et l'intensité de leurs réponses leur ont permis de se faire prendre au sérieux par les hommes et les femmes de leur communauté.

9. Les gains pratiques et à court-terme acquis par les femmes peuvent être complémentés par des changements planifiés stratégiquement sur plusieurs générations. A court-terme, les projets d'aménagement des terres incultes ont conféré aux femmes le droit d'exploiter collectivement des terres communales. Ceci peut être vu comme une première étape pouvant éventuellement déboucher sur l'autorisation pour les femmes de posséder leurs propres terres-ce qui est très rare en Inde. Plusieurs membres du groupe ont déjà formulé le souhait qu'après leur mort, les filles aient droit aux mêmes parts d'héritage que les garçons. C'est à travers de tels modes de pensée stratégiques qu'il sera possible de modifier à long-terme les pratiques qui perpétuent le caractère subalterne de la condition de la femme.

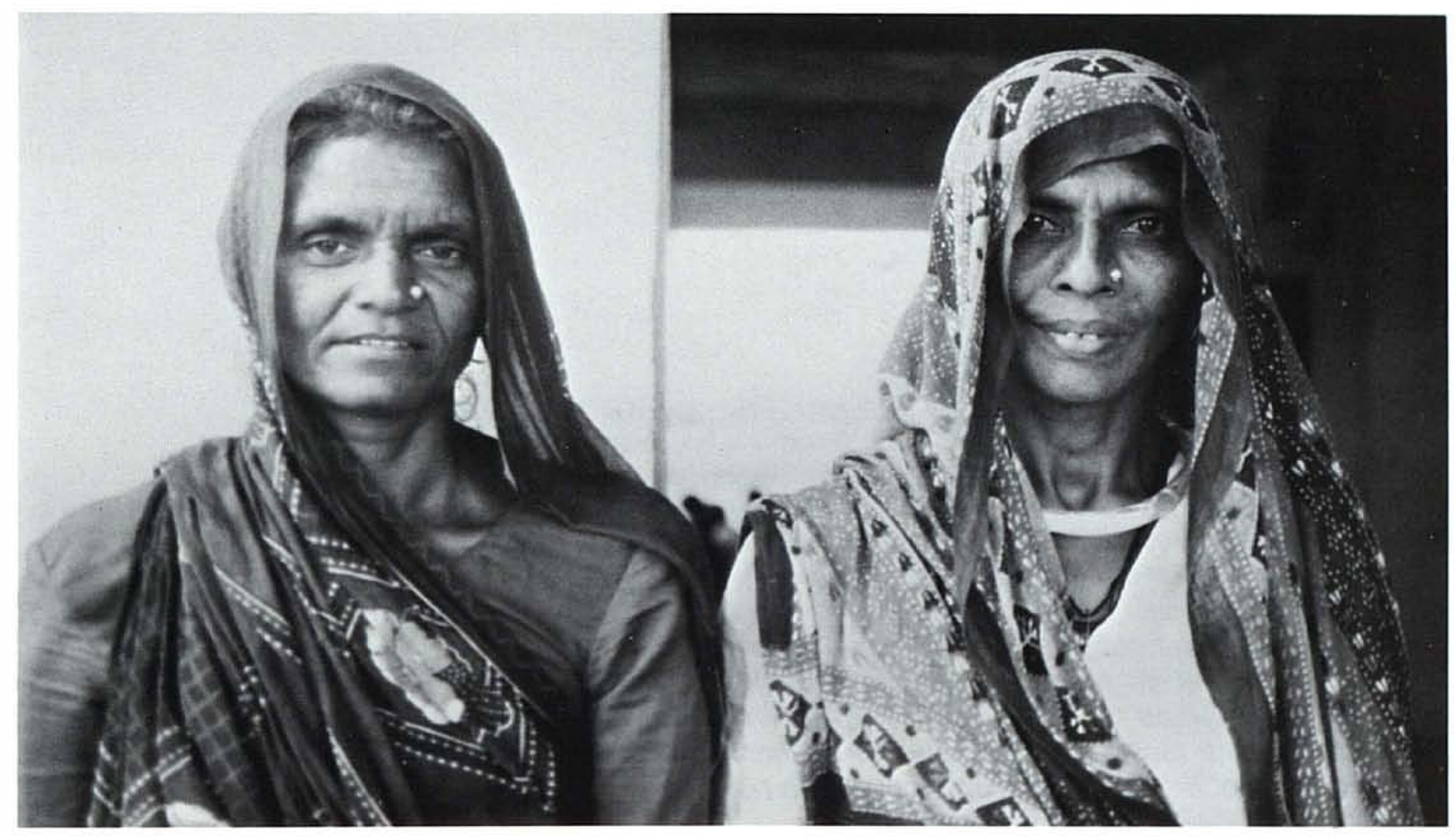




\section{Annexe}

Ci-dessous, figurent quelques publications consacrées au thème de l'environnement et de l'émancipation des femmes. Pour obtenir des renseignements sur la disponibilité, le coût et les frais de port, veuillez contacter directement la maison d'édition indiquée entre parenthèses.

Asia \& Pacific Women's Resource and Action Series: Environment, (APDC, P.O. Box 12224, 50770 Kuala Lumpur, Malaisie, 1992)

Cold Hearths and Barren Slopes, the Wood Fuel Crisis in the Third World, par Bina Agarwal (ZED Books, 57 Caledonian Road, Londres N1 9BU, Angleterre, 1988)

"Community Management of Waste Recycling: The SIRDO", par Marianne Schmink, disponbile en anglais et en espagnol (SEEDS, P.O. Box 3923, Grand Central Station, New York, N.Y. 10163, Etats-Unis d'Amérique, 1984)

"La Conservation des Forêts au Népal: Encourager la Participation des Femmes", par Augusta Molnar, disponible en français, en anglais et en espagnol (SEEDS, P.O. Box 3923, Grand Central Station, New York, N.Y. 10163, Etats-Unis d'Amérique, 1987)

"Gender Planning in the Third World: Meeting Practical and Strategic Gender Needs", par Caroline O.N. Moser (World Development, Vol. 17, no. 11, 1989)

Women and the Environment: A Reader, Sally Sontheimer, ed. (Monthly Review Foundation, 122 West 27th Street, New York, N.Y. 10001, Etats-Unis d'Amérique, 1991)

The Bankurra Story: Rural Women Organise for Change and Technical Cooperation, rapport, par Nalini Singh (ILO, World Employment Branch, New Delhi, Inde, 1988)

"Women, Environment and Development", The Tribune, Newsletter 47, septembre 1991 (ITWC, 777 United Nations Plaza, New York, N.Y. 10017, Etats-Unis d'Amérique)

Madhu Sarin a travaillé en collaboration avec SARTHI et PEDO depuis 1983 d'abord à titre individuel puis comme membre d'une petite équipe de spécialistes des questions de femmes. Elle aimerait exprimer sa reconnaissance:

- aux membres des tous les groupes de femmes pour tout le temps qu'elles ont bien voulu lui accorder et pour partager leurs expériences associatives;

- au directeur et au personnel de terrain de SARTHI pour partager leurs observations et leurs connaissances; et

- à ses chères amies et collègues, Renu Khanna et Chandrika Sharma, avec lesquelles elle a travaillé depuis qu'elles ont commencé à se pencher sur la question du rôle des femmes rurales dans la gestion des ressources locales. 
Mise en page: Ann Leonard

Typographie: Village Type and Graphics

Photos: Madhu Sarin

Imprimeur: Graphic Impressions, Inc.

Traduction: Pia Heitz

\section{Les Numéros de SEEDS Disponsibles en Français}

No. 3 "Les Coopératives des Vendeuses de Marché: Accorder Crédit aux Femmes" par Judith Bruce (Nicaragua)

No. 4 "Les Femmes et L'Artisanat: Mythe et Réalité" par Jasleen Dhamija (International)

No. 5 "La Coopérative de Markala: Une Nouvelle Approche des Rôles Economique Traditionnaux" par Susan Caughman et Mariam N'diaye Thaim (Sénégal)

No. 6 "Le Forum pour les Femmes Actives: Lutte pour le Crédit et le Changement"' par Marty Chen (Inde)

No. 7 "Creér des Emplois pour Les Femmes dans des Activités de Production Non-Artisanales au Bangladesh" par Marty Chen (Bangladesh)

No. 10 "La Conservation des Forêts au Népal: Encourager la Participation des Femmes" par Augusta Molnar (Nepal)

No. 14 "Faire Oeuvre de Poinnier: Atteindre les Femmes Agriculteurs en Zambie Occidentale" par Janice Jiggins avec Paul Maimbo et Mary Masona (Zambie)

Nous attendons vos remarques, commentaires et idées de projets à publier dans les prochains numéros de SEEDS. Si vous souhaitez recevoir des exemplaires supplémentaires de ce numéro ou faire partie de nos abonnés, n'hésitez pas à nous écrire. Envoyez votre courrier à:

Ann Leonard, Conseillère à la Rédaction

$$
\text { SEEDS }
$$

P.O. Box 3923

Grand Central Station

New York, New York 10163 U.S.A. 


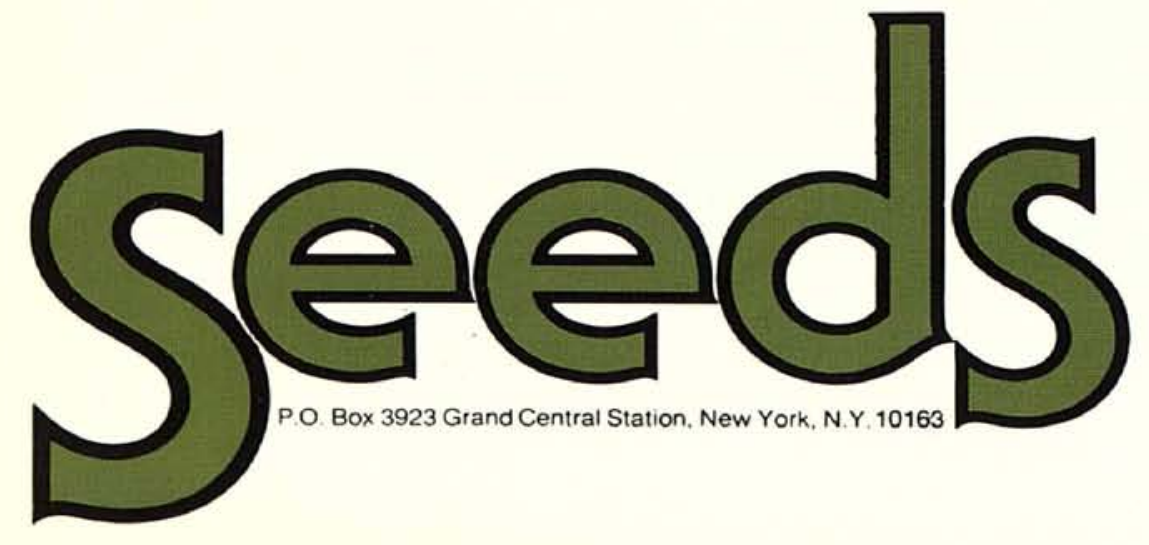

